\title{
DISPERSION VS. ANTI-DIFFUSION: WELL-POSEDNESS IN VARIABLE COEFFICIENT AND QUASILINEAR EQUATIONS OF KDV-TYPE.
}

\author{
DAVID M. AMBROSE AND J. DOUGLAS WRIGHT
}

\begin{abstract}
We study the well-posedness of the initial value problem on periodic intervals for linear and quasilinear evolution equations for which the leading-order terms have three spatial derivatives. In such equations, there is a competition between the dispersive effects which stem from the leadingorder term, and anti-diffusion which stems from the lower-order terms with two spatial derivatives. We show that the dispersive effects can dominate the backwards diffusion: we find a condition which guarantees well-posedness of the initial value problem for linear, variable coefficient equations of this kind, even when such anti-diffusion is present. In fact, we show that even in the presence of localized backwards diffusion, the dispersion will in some cases lead to an overall effect of parabolic smoothing. By contrast, we also show that when our condition is violated, the backwards diffusion can dominate the dispersive effects, leading to an ill-posed initial value problem. We use these results on linear evolution equations as a guide when proving well-posedness of the initial value problem for some quasilinear equations which also exhibit this competition between dispersion and anti-diffusion: a Rosenau-Hyman compacton equation, the Harry Dym equation, and equations which arise in the numerical analysis of finite difference schemes for dispersive equations. For these quasilinear equations, the well-posedness theorem requires that the initial data be uniformly bounded away from zero.
\end{abstract}

\section{Introduction}

We study a family of evolution equations,

$$
u_{t}=a u_{x x x}+b u_{x x}+\phi,
$$

where $a$ and $b$ are functions and $\phi$ represents a collection of lower-order terms, and where $x \in X=[0, M]$ and we assume $u$ satisfies periodic boundary conditions. We establish conditions on the coefficient functions $a$ and $b$, as well as conditions on $\phi$, which ensure that the initial value problem for this equation is well-posed in Sobolev spaces. This is a delicate question because if there exist any $x$ and $t$ for which $b<0$, then formally, this term acts (locally, at least) as an anti-diffusion or backwards heat operator. The conditions we find on $a$ and $b$ indicate to what extent the dispersive effects of the leading-order term can compensate for the presence of this anti-diffusion. In a special case (in which the coefficient functions depend only on $x$ ), we also show that if our condition is violated, then the initial value problem is ill-posed. We treat this problem for linear equations, for which $a$ and $b$ are functions of $x$ and $t$, and also certain quasilinear equations, for which $a$ and $b$ depend on $u$ and $u_{x}$. 
Our original motivation for this work is to study the question of well-posedness or ill-posedness for the the Rosenau-Hyman $K(2,2)$ equation,

$$
u_{t}=2 u u_{x x x}+6 u_{x} u_{x x}+2 u u_{x} .
$$

$K(2,2)$ is a degenerate version of the Korteweg-de Vries equation and famously supports compactly supported traveling waves, dubbed compactons [15, as well as other non-analytic traveling waves [13. Many papers in the literature make numerical simulations of solutions of the $K(2,2)$ initial value problem, focusing primarily on the study of interactions of these compactons [4, 8], 12], [16], [17. While the existence theory for this equation remains unsettled, there is strong evidence that $K(2,2)$ is ill-posed for data which crosses zero [2]. Here we study a complementary problem: Is the equation well-posed when the initial condition is bounded away from zero? The answer, as we show below, is yes.

In order to show that $K(2,2)$ is well-posed, we first study an associated linear problem:

$$
u_{t}=a(x, t) u_{x x x}+b(x, t) u_{x x}+c(x, t) u_{x}+d(x, t) u+e(x, t)
$$

where once again $x \in X=[0, M]$ and $u$ (and the coefficient functions) satisfy periodic boundary conditions. We assume that the coefficient functions are defined for all $t \in[0, T]$, where $T>0$.

By way of analogy, supposing that $u(x, 0)$ is bounded away from zero in $K(2,2)$ we should study the situation where $a(x, t)$ is likewise bounded from zero. On the other hand, $u_{x}(x, 0)$ will change signs for any non-trivial periodic initial data and so we should not impose strong conditions on $b(x, t)$. In particular we must allow $b(x, t)$ to be negative, at least for some $x$.

Equations like (2), and quasilinear variants, have been studied previously, though the focus has been on the case where $X=\mathbf{R}$ instead of the periodic problem we study here. The earliest work [6] requires that $b \geq 0$; that is to say, there is no destabilizing "backwards diffusion" effect from the term $b(x, t) u_{x x}$. It is wellknown that if $a(x, t)$ is identically zero and there is an interval on which $b<0$, then these destabilizing effect are so pathological that (2) is ill-posed for data in Sobolev spaces. In some sense, the results of this paper are an investigation into the extent to which dispersive effects from $a(x, t) u_{x x x}$ ameliorate the catastrophically destabilizing effects caused by a backwards diffusive term.

This question was answered for $X=\mathbf{R}$ in [1], and, in short, the answer is that if $b(\cdot, t) \in L^{1}$ uniformly in $t$ and $a(x, t)$ is bounded away from zero, then the dispersive effects completely dominate the backwards diffusion and the equation (including quasilinear versions) is well-posed. The issues for the periodic setting are necessarily different; the argument cannot be based on integrability (the integrability would be considered over the real line rather than a periodic interval, and a nontrivial periodic function is not integrable on the real line). The answers that we find for the periodic case include a well-posedness theorem, a parabolic smoothing result, and an ill-posedness theorem for (2), when certain conditions (primarily on $a$ and b) are satisfied.

While we were motivated by the well-posedness question for the $K(2,2)$ equation, our method of proof applies to other quasilinear evolution equations as well. We do not attempt to give the most general theorem that we can on well-posedness of quasilinear evolution equations with nonlinear dispersion and anti-diffusion, but 
instead we consider some additional equations which have appeared in the literature. One of these is the Harry Dym equation, $u_{t}=u^{3} u_{x x x}$. Other examples come from the numerical analysis of finite difference schemes for certain dispersive partial differential equations. We are able to prove well-posedness theorems for such equations for uniformly positive Sobolev initial data.

The remainder of this paper is organized as follows. In Section 2 we study the linear problem (2) and provide sufficient conditions on the coefficients so that the equation is well-posed. The main tools here are a change of dependent variables followed by energy estimates. See Theorem 1 below for the precise statement of this result. In Section 3 we use the linear energy estimates as a inspiration for developing analogous estimates for $K(2,2)$. These estimates are sufficient for proving that $K(2,2)$ is well-posed. See Corollary 19 below for the precise statement of this result. In Section 4, we briefly discuss additional quasilinear equations which can be shown to be well-posed for positive initial data in Sobolev spaces. We mention that all of our well-posedness proofs largely follow the lines of Chapter 3 of [14], in which the Navier-Stokes equations are shown to be well-posed in Sobolev spaces. In Section 5, we give a theorem on singularity formation: if positive solutions of these quasilinear equations ever touch down and obtain a value of zero, then the solutions must blow up in the space $H^{4}$.

\section{THE LINEAR PROBLEM}

2.1. Main ideas and instructive examples. The following heuristics and special cases provide some crucial ideas for how we proceed. The first special case we consider is

$$
u_{t}=a_{0} u_{x x x}-b_{0} u_{x x} \quad \text { with } \quad x \in[0,2 \pi]
$$

where $a_{0}$ and $b_{0}$ are positive and constant and $u$ satisfies periodic boundary conditions. The equation can be solved via Fourier series:

$$
u(x, t)=\sum_{k \in \mathbf{Z}} \exp \left(-i a_{0} k^{3} t\right) \exp \left(b_{0} k^{2} t\right) e^{i k x} \widehat{u}(k, 0) .
$$

Here $\widehat{u}(k, 0)$ are the coefficients of the Fourier series expansion of the initial data. A routine calculation shows that the right hand side of this equation, viewed as a map from $\mathbf{R}^{+} \times H^{s}$ into $H^{s}$ does not depend continuously on $u(x, 0)$. This is due to the fact that $\exp \left(b_{0} k^{2} t\right)$ grows extremely fast for large wave numbers $k$. As such, (3) is ill-posed in precisely the same way that backwards heat equation is.

If $b$ is uniformly negative, this example illustrates that there is no chance for the dispersive effects to play a role (above, we had $b(x, t)=-b_{0}<0$ ). However, if $b$ is localized and negative, there is reason to think that the dispersion can arrest blow up, as the following formal heuristic demonstrates. Specifically, consider the following:

$$
u_{t}=a_{0} u_{x x x}+b(x) u_{x x} \quad \text { with } \quad x \in[-M, M]
$$

where

$$
b(x)= \begin{cases}-b_{0}<0, & \text { for } x \in[0, L] \\ 0, & \text { otherwise }\end{cases}
$$

Here $0<L \ll M$. For simplicity, assume $a_{0}<0$. Consider a "wave packet" of amplitude $\mu_{0}$ and wave number $k_{0}$ which is initially located to the left of $x=0$. Such 


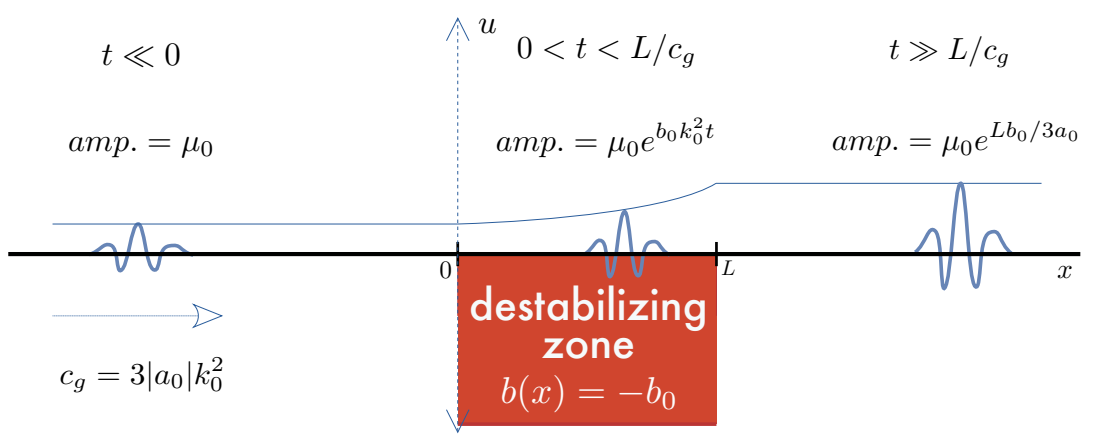

Figure 1. Dispersion vs localized backwards diffusion in (4).

a wave packet will move with group speed associated to the equation $u_{t}=a_{0} u_{x x x}$, which is to say with speed

$$
c_{g}=3\left|a_{0}\right| k_{0}^{2}
$$

When the packet enters the interval $[0, L]$, say at $t=0$, it will be subjected to the effects of the backwards heat term, which will cause the amplitude to grow like

$$
\mu_{0} \exp \left(b_{0} k_{0}^{2} t\right)
$$

as was the case in (3). The wave packet will leave the interval $[0, L]$ at time $t_{\text {exit }}=L / c_{g}$ and thus the final amplitude of the packet is

$$
\mu_{0} \exp \left(b_{0} k_{0}^{2} t_{\text {exit }}\right)=\mu_{0} \exp \left(b_{0} k_{0}^{2} \frac{L}{3\left|a_{0}\right| k_{0}^{2}}\right)=\mu_{0} \exp \left(\frac{L}{3}\left|\frac{b_{0}}{a_{0}}\right|\right) .
$$

See Figure 1. The growth of the amplitude during the wave packet's passage through the destabilizing zone is independent of its wave number - the extremely rapid growth of high wave numbers is held in check by the fact that these highly oscillatory wave packets move very fast.

Therefore, if we presume that $b(x, t)$ is not everywhere negative, this heuristic indicates a plausible mechanism by which dispersion can arrest blowup. Note that equation (5) points to the importance of the ratio

$$
\delta(x, t):=\frac{b(x, t)}{|a(x, t)|}
$$

in determining the growth of the amplitude. We call $\delta(x, t)$ the modified diffusion for the equation, for reasons which will become clear below.

We make this formal argument - which is closely related to the geometric optics heuristics described in 7]-precise in this article. Specifically, we give sufficient conditions on the coefficient functions $a(x, t), \ldots, e(x, t)$ so that 22 is well-posed. In what follows $W^{k, p}=W^{k, p}(X)$ is the usual Sobolev space of periodic functions on $X$, and $H^{n}=H^{n}(X)=W^{n, 2}$. Also, for any Banach space of functions defined on $X$, we set

$$
B_{T}:=L^{\infty}([0, T] ; B) .
$$

We now state our assumptions on the coefficient functions:

Assumption 1. The coefficients are sufficiently regular/integrable: Fix $n \in \mathbf{N}$, with $n \geq 4$. Then

$$
\text { - } a(x, t) \in C\left([0, T] ; C^{n+3}\right) \text {, }
$$


- $a_{t}(x, t) \in C\left([0, T] ; C^{n}\right)$,

- $b(x, t) \in C\left([0, T] ; C^{n+2}\right)$,

- $b_{t}(x, t) \in C\left([0, T] ; C^{n}\right)$,

- $c(x, t) \in C\left([0, T] ; C^{n}\right)$,

- $d(x, t) \in C\left([0, T] ; C^{n}\right)$, and

- $e(x, t) \in L^{\infty}\left([0, T] ; H^{n}\right)$.

Assumption 2. The dispersion does not degenerate: There exists a constant $a_{0}$ with the property that for all $x \in X$ and $t \in[0, T]$ :

$$
0<a_{0} \leq|a(x, t)| .
$$

Assumption 3. The average modified diffusion is non-negative:

$$
\bar{\delta}(t):=\frac{1}{M} \int_{0}^{M} \frac{b(y, t)}{|a(y, t)|} d y \geq 0
$$

for all $t \in[0, T]$.

Remark 1. Let

$$
\begin{aligned}
\tilde{K}:=M+T+n+\|a\|_{W_{T}^{n+3, \infty}}+\left\|a_{t}\right\|_{W_{T}^{1, \infty}}+\left\|a^{-1}\right\|_{L_{T}^{\infty}} \\
+\|b\|_{W_{T}^{n, \infty}}+\left\|b_{t}\right\|_{L_{T}^{\infty}}+\|c\|_{W_{T}^{n, \infty}}+\|d\|_{W_{T}^{n, \infty}}+\|e\|_{H_{T}^{n}} .
\end{aligned}
$$

Throughout this Section, any constant $K$ which appears in an estimate has the property that $K>0$ and $K$ depends only on $\tilde{K}$. For instance, one can show that

$$
\bar{\delta}(t)+\left|\frac{d}{d t} \bar{\delta}(t)\right| \leq K .
$$

Our first main result is:

Theorem 1. Fix $n \in \mathbf{N}$ such that $n \geq 4$ and take Assumptions 1 , 2 and 3 to be true. Then there is a continuous map

$$
\Psi: H^{n} \longrightarrow L^{\infty}\left([0, T] ; H^{n}\right)
$$

such that for all $u_{0} \in H^{n}$,

$$
\left\|\Psi\left(u_{0}\right)\right\|_{L^{\infty}\left([0, T] ; H^{n}\right)} \leq K\left(\left\|u_{0}\right\|_{H^{n}}+1\right),
$$

and such that the following are true for $u=\Psi\left(u_{0}\right)$ :

(1) For all $0 \leq n^{\prime}<n, u \in C\left([0, T] ; H^{n^{\prime}}\right)$ and $\|u(t)\|_{H^{n^{\prime}}} \leq K\left(\left\|u_{0}\right\|_{H^{n}}+1\right)$,

(2) $u(x, 0)=u_{0}$,

(3) $u(x, t)$ solves $(2)$ for $t \in[0, T]$ and

(4) $u(x, t)$ is the only function for which (2) and (3) hold simultaneously.

We have two extensions of this if we have more information about $\bar{\delta}(t)$. First,

Theorem 2. If, in addition to Assumptions 1. 2 and [3, we have for all $t \in[0, T]$

$$
\bar{\delta}(t) \geq \delta_{0}>0
$$

then the solution map $\Psi$ from Theorem 1 enjoys a parabolic smoothing property. That is, for all $u_{0} \in H^{n}$,

$$
\left\|\Psi\left(u_{0}\right)\right\|_{L^{2}\left([0, T] ; H^{n+1}\right)} \leq K\left\|u_{0}\right\|_{H^{n}} .
$$

Moreover, if Assumption 1 holds with $n$ replaced by $n+1$, then $u=\Psi\left(u_{0}\right) \in$ $C\left([0, T] ; H^{n}\right) \cap C\left((0, T] ; H^{n}\right)$ for all $n^{\prime} \in[0, n+1)$. 
Theorem 3. If, in addition to Assumptions 1. 2 and 3, we have for all $t \in[0, T]$

$$
\bar{\delta}(t)=0,
$$

then then the solution map $\Psi$ from Theorem 1 satisfies $u=\Psi\left(u_{0}\right) \in C\left([0, T] ; H^{n}\right)$.

Remark 2. To demonstrate how surprising our results are, consider the following examples, all posed for $x \in[0,2 \pi]$. Clearly, the initial value problem for the equation

$$
u_{t}=\sin (x) u_{x x}
$$

is ill-posed. However, if we add even a very small amount of dispersion, for instance,

$$
u_{t}=\frac{1}{10^{23}} u_{x x x}+\sin (x) u_{x x}
$$

then the initial value problem for the equation is well-posed since the average modified diffusion in this case is equal to zero ( $\operatorname{since} \sin (x)$ has average value zero).

Similarly, for any $\varepsilon$ such that $0<\varepsilon<1$, the initial value problem for the equation

$$
u_{t}=(\varepsilon+\sin (x)) u_{x x}
$$

is again ill-posed. However, if we consider the equation with some dispersion, say

$$
u_{t}=\frac{1}{10^{23}} u_{x x x}+(\varepsilon+\sin (x)) u_{x x},
$$

then since $\bar{\delta}=10^{23} \varepsilon>0$, the initial value problem is well-posed. Morever, Theorem 2 states that even for $a(x, t)$ ludicrously small, as in this case, so long as $\varepsilon>0$, the equation is essentially parabolic even though, on a sizable fraction of the interval, there is a backwards heat operator acting.

Remark 3. One can ask which of the assumptions are necessary as well as sufficient. We do not have definitive answers at this time, but some relevant information is known. The results in [2] and [7] strongly indicate that if $a(x, t)$ crosses zero for some $x$ with non-zero derivative, then (2) will be ill-posed. The regularity/integrability conditions in Assumption 1 can almost certainly be weakened, but doing so is likely technical and not terribly interesting at this time. A quick calculation shows that sending $t \rightarrow-t$ in (2) results in an equation of the same form as $(2)$ but reverses the sign of $\bar{\delta}$. The parabolic smoothing property in Theorem 2 indicates that it is highly unlikely that (2) would be well-posed backwards in time when $\bar{\delta}(t)>0$. These two facts together indicate that Assumption 3 is almost certainly necessary. We confirm this in the special case when the coefficients functions are time independent in Theorem 10 below.

Remark 4. Assumption 2 allows us, without loss of generality, to take

$$
a(x, t) \geq a_{0}>0 .
$$

And so, from this point on, $a(x, t)$ is presumed to be positive.

2.2. The change of variables and key estimate. Our goal is to find an a priori estimate for solutions $u(x, t)$ of $(2)$ in the space $H^{n}$. It turns out that it this more easily done after changing dependent variables. Toward this end, we let $g_{n}(x, t)$ be function which has the following three properties:

(C1) There is a constant $k_{g}>1$ such that for all $x$ and $t$,

$$
k_{g}^{-1} \leq g_{n}(x, t) \leq k_{g}
$$

$(\mathrm{C} 2) g_{n} \in C\left([0, T] ; C^{n+3}\right)$ and $\partial_{t} g_{n} \in C\left([0, T] ; C^{n}\right)$, and 
(C3) $g_{n}$ and its first $n$ derivatives satisfy periodic boundary conditions.

Then define $v(x, t)$ via

$$
u(x, t):=g_{n}(x, t) v(x, t) .
$$

An intelligent choice for $g_{n}$ will allow us to find an energy estimate for $v$, and we will make such a choice at the appropriate place in our proof. The following Lemma, whose proof is obvious, implies we will have a comparable estimate for $u$.

Lemma 4. Assume $g_{n}$ satisfies (C1)-(C3). Then $u \in H_{T}^{n}$ if and only if $v \in H_{T}^{n}$. That is, there is a constant $C_{g} \geq 1$, depending only on $\left\|g_{n}\right\|_{W_{T}^{n, \infty}}$ and $\left\|g_{n}^{-1}\right\|_{L_{T}^{\infty}}$, such that

$$
C_{g}^{-1}\|u\|_{H_{T}^{n}} \leq\|v\|_{H_{T}^{n}} \leq C_{g}\|v\|_{H_{T}^{n}} .
$$

Substituting (6) into (2) yields the following after a routine calculation:

$$
v_{t}=a v_{x x x}+\tilde{b} v_{x x}+\tilde{c} v_{x}+\tilde{d} v+\tilde{e}
$$

where

$$
\begin{aligned}
& \tilde{b}(x, t)=3 a \frac{\partial_{x} g_{n}}{g_{n}}+b \\
& \tilde{c}(x, t)=3 a \frac{\partial_{x}^{2} g_{n}}{g_{n}}+2 b \frac{\partial_{x} g_{n}}{g_{n}}+c \\
& \tilde{d}(x, t)=-\frac{\partial_{t} g_{n}}{g_{n}}+a \frac{\partial_{x}^{3} g_{n}}{g_{n}}+b \frac{\partial_{x}^{2} g_{n}}{g_{n}}+c \frac{\partial_{x} g_{n}}{g_{n}}+d \\
& \tilde{e}(x, t)=\frac{e}{g_{n}} .
\end{aligned}
$$

Remark 5. The regularity assumptions in $(\mathrm{C} 2)$ are made so that $\tilde{b}, \tilde{c}, \tilde{d}$ and $\tilde{e}$ are in the same spaces as $b, c, d$ and $e$.

Note that the right hand side of (7) is of the same form as that of (2). Motivated by this, define the operator

$$
L_{A B C D}:=A \partial_{x}^{3}+B \partial_{x}^{2}+C \partial_{x}+D
$$

where $A, B, C$ and $D$ are functions which satisfy periodic boundary conditions in $x$. The following Lemma contains the heart of our energy estimate:

Lemma 5. Fix $n \in \mathbf{N}$ with $n \geq 4$. Suppose that $A, B, C, D \in W^{n, \infty}$. Then there exists $\kappa>0$ (which depends only on $n, M$ and the $W^{n, \infty}$ norms of $A, B, C$ and $D$ ) such that, for all $\phi \in H^{n}$ :

$$
\int_{X}\left(\partial_{x}^{n} \phi\right) \partial_{x}^{n}\left(L_{A B C D} \phi\right) d x-\int_{X}\left[\left(\frac{3}{2}-n\right) A_{x}-B\right]\left[\partial_{x}^{n+1} \phi\right]^{2} d x \leq \kappa\|\phi\|_{H^{n}}^{2} .
$$

Proof: We prove the result for smooth functions $\phi$ only. The case for $\phi \in H^{n}$ follows by a typical density argument. From the defintion of $L_{A B C D}$, we have:

$$
\begin{aligned}
\int_{X}\left(\partial_{x}^{n} \phi\right) \partial_{x}^{n}\left(L_{A B C D} \phi\right) d x & =\int_{X}\left(\partial_{x}^{n} \phi\right) \partial_{x}^{n}\left(A \phi_{x x x}+B \phi_{x x}+C \phi_{x}+D \phi\right) d x \\
& =I_{A}+I_{B}+I_{C}+I_{D}
\end{aligned}
$$

where $I_{A}, I_{B}, I_{C}$ and $I_{D}$ are defined in the obvious way. We estimate each in turn. 
First, the product rule gives:

$$
\begin{aligned}
I_{A}:= & \int_{X}\left(\partial_{x}^{n} \phi\right) \partial_{x}^{n}\left(A \phi_{x x x}\right) d x \\
= & \int_{X}\left(\partial_{x}^{n} \phi\right) A \partial_{x}^{n+3} \phi d x+\int_{X} n\left(\partial_{x}^{n} \phi\right) A_{x} \partial_{x}^{n+2} \phi d x \\
& +\int_{X}\left(\begin{array}{c}
n \\
2
\end{array}\right)\left(\partial_{x}^{n} \phi\right) A_{x x} \partial_{x}^{n+1} \phi d x+\sum_{j=0}^{n-3} \int_{X}\left(\begin{array}{c}
n \\
j
\end{array}\right)\left(\partial_{x}^{n} \phi\right)\left(\partial_{x}^{n-j} A\right) \partial_{x}^{j+3} \phi d x .
\end{aligned}
$$

We call the four terms on the right hand side $I_{A}^{1}, I_{A}^{2}, I_{A}^{3}, I_{A}^{4}$ respectively.

$I_{A}^{4}$ is controlled with the Cauchy-Schwarz inequality and other elementary tools, since at most $n$ derivatives of $\phi$ appear:

$$
I_{A}^{4}:=\sum_{j=0}^{n-3} \int_{X}\left(\begin{array}{c}
n \\
j
\end{array}\right)\left(\partial_{x}^{n} \phi\right)\left(\partial_{x}^{n-j} A\right) \partial_{x}^{j+3} \phi d x \leq \kappa\|\phi\|_{H^{n}}^{2} .
$$

$I_{A}^{3}$ is handled using the observation that $\partial_{x}^{n} \phi \partial_{x}^{n+1} \phi=\frac{1}{2} \partial_{x}\left[\partial_{x}^{n} \phi\right]^{2}$. Thus an integration by parts gives:

$$
\begin{aligned}
I_{A}^{3}:=\int_{X}\left(\begin{array}{l}
n \\
2
\end{array}\right)\left(\partial_{x}^{n} \phi\right) A_{x x} \partial_{x}^{n+1} \phi d x & =\frac{1}{2}\left(\begin{array}{l}
n \\
2
\end{array}\right) \int_{X} A_{x x} \partial_{x}\left[\partial_{x}^{n} \phi\right]^{2} d x \\
& =-\frac{1}{2}\left(\begin{array}{l}
n \\
2
\end{array}\right) \int_{X} A_{x x x}\left[\partial_{x}^{n} \phi\right]^{2} d x \leq \kappa\|\phi\|_{H^{n}}^{2} .
\end{aligned}
$$

A quick calculation shows that, for any smooth function $\psi$,

$$
\psi \psi_{x x x}=\frac{1}{2} \partial_{x}^{3}\left[\psi^{2}\right]-\frac{3}{2} \partial_{x}\left[\psi_{x}\right]^{2} .
$$

Applying this with $\psi=\partial_{x}^{n} \phi$ in $I_{A}^{1}$ gives:

$$
I_{A}^{1}:=\int_{X} A\left(\partial_{x}^{n} \phi\right) \partial_{x}^{n+3} \phi d x=\frac{1}{2} \int_{X} A \partial_{x}^{3}\left[\partial_{x}^{n} \phi\right]^{2} d x-\frac{3}{2} \int_{X} A \partial_{x}\left[\partial_{x}^{n+1} \phi\right]^{2} d x .
$$

Integrating by parts three times in the first term and just once in the second term gives:

$$
I_{A}^{1}=-\frac{1}{2} \int_{X} A_{x x x}\left[\partial_{x}^{n} \phi\right]^{2} d x+\frac{3}{2} \int_{X} A_{x}\left[\partial_{x}^{n+1} \phi\right]^{2} d x \leq \frac{3}{2} \int_{X} A_{x}\left[\partial_{x}^{n+1} \phi\right]^{2} d x+\kappa\|\phi\|_{H^{n}}^{2} .
$$

Now we consider

For smooth functions $\psi$, we have

$$
I_{A}^{2}:=\int_{X} n A_{x}\left(\partial_{x}^{n} \phi\right) \partial_{x}^{n+2} \phi d x
$$

$$
\psi \psi_{x x}=\frac{1}{2} \partial_{x}^{2}\left[\psi^{2}\right]-\left[\psi_{x}\right]^{2} .
$$

If we use this with $\psi=\partial_{x}^{n} \phi$, then

$$
I_{A}^{2}=\frac{1}{2} \int_{X} n A_{x} \partial_{x}^{2}\left[\partial_{x}^{n} \phi\right]^{2} d x-\int_{X} n A_{x}\left[\partial_{x}^{n+1} \phi\right]^{2} d x
$$

Integrating by parts twice in the first term gives:

$I_{A}^{2}=\frac{1}{2} \int_{X} n A_{x x x}\left[\partial_{x}^{n} \phi\right]^{2} d x-\int_{X} n A_{x}\left[\partial_{x}^{n+1} \phi\right]^{2} d x \leq-\int_{X} n A_{x}\left[\partial_{x}^{n+1} \phi\right]^{2} d x+\kappa\|\phi\|_{H^{n}}^{2}$. 
Adding the estimates for $I_{A}^{1}, I_{A}^{2}, I_{A}^{3}$ and $I_{A}^{4}$ yields:

$$
I_{A} \leq\left(\frac{3}{2}-n\right) \int_{X} A_{x}\left[\partial_{x}^{n+1} \phi\right]^{2} d x+\kappa\|\phi\|_{H^{n}}^{2} .
$$

Now we estimate $I_{B}$. As with $I_{A}$, the product rule gives:

$$
\begin{aligned}
I_{B}:= & \int_{X}\left(\partial_{x}^{n} \phi\right) \partial_{x}^{n}\left(B \phi_{x x}\right) d x \\
= & \int_{X}\left(\partial_{x}^{n} \phi\right) B \partial_{x}^{n+2} \phi d x+\int_{X} n\left(\partial_{x}^{n} \phi\right) B_{x} \partial_{x}^{n+1} \phi d x \\
& +\sum_{j=0}^{n-2} \int_{X}\left(\begin{array}{c}
n \\
j
\end{array}\right)\left(\partial_{x}^{n} \phi\right)\left(\partial_{x}^{n-j} B\right) \partial_{x}^{j+2} \phi d x .
\end{aligned}
$$

Using the same steps as in 111$)$ and $(12)$, we have

$$
\int_{X} n\left(\partial_{x}^{n} \phi\right) B_{x} \partial_{x}^{n+1} \phi d x+\sum_{j=0}^{n-2} \int_{X}\left(\begin{array}{c}
n \\
j
\end{array}\right)\left(\partial_{x}^{n} \phi\right)\left(\partial_{x}^{n-j} B\right) \partial_{x}^{j+2} \phi d x \leq \kappa\|\phi\|_{H^{n}}^{2} .
$$

Notice that the other term in $I_{B}$, namely $\int_{X} B\left(\partial_{x}^{n} \phi\right) \partial_{x}^{n+2} \phi d x$, is the same as $I_{A}^{2}$ but with $B$ replacing $n A_{x}$. So using (13) and following the steps used to estimate $I_{A}^{2}$ we get:

$$
\int_{X} B\left(\partial_{x}^{n} \phi\right) \partial_{x}^{n+2} \phi d x \leq-\int_{X} B\left[\partial_{x}^{n+1} \phi\right]^{2} d x+\kappa\|\phi\|_{H^{n}}^{2} .
$$

Combining these estimates, we have

$$
I_{B} \leq-\int_{X} B\left[\partial_{x}^{n+1} \phi\right]^{2} d x+\kappa\|\phi\|_{H^{n}}^{2} .
$$

Controlling $I_{C}$ and $I_{D}$ amounts to applying the processes in 11 and 12 appropriately. One finds

$$
I_{C}+I_{D} \leq \kappa\|\phi\|_{H^{n}}^{2} .
$$

Adding this to 14 and 15 then rearranging terms in 10 completes the proof of the lemma.

2.3. Selection of $g_{n}$. Notice that if $\left(\frac{3}{2}-n\right) A_{x}-B \leq 0$, that the second term on the left hand side of $(9)$ is non-negative, so dropping it gives

$$
\int_{X}\left(\partial_{x}^{n} \phi\right) \partial_{x}^{n}\left(L_{A B C D} \phi\right) d x \leq \kappa\|\phi\|_{H^{n}}^{2} .
$$

This fact will lead to an a priori $H^{n}$ estimate for solutions $v$ of $(7)$ provided

$$
\left(\frac{3}{2}-n\right) a_{x}-\tilde{b} \leq 0
$$

Given the definition of $\tilde{b}$ this is the same as requiring that the following differential inequality is satisfied:

$$
\left(\frac{3}{2}-n\right) a_{x}-b-3 a \frac{\partial_{x} g_{n}}{g_{n}} \leq 0 \quad \text { for all } x \in X \text { and } t \in[0, T] .
$$


At first glance, the most straightforward way to meet 17 is to choose $g_{n}$ so that that $\left(\frac{3}{2}-n\right) a_{x}-b-3 a \frac{\partial_{x} g_{n}}{g_{n}}=0$. That is to say, take $g_{n}$ to be

$$
(a(x, t))^{1 / 2-n / 3} \exp \left[-\frac{1}{3} \int_{0}^{x} \frac{b(y, t)}{a(y, t)} d y\right] .
$$

The problem with doing this is the fact that this function does not meet periodic boundary conditions, as $\int_{0}^{M} \frac{b(y, t)}{a(y, t)} d y$ is not necessarily zero. Since our estimates require multiple integrations by parts, it is absolutely critical that $g_{n}$ be periodic. Assumption 3 is made precisely so that we may choose $g_{n}$ to be periodic while simultaneously satisfying (17). (It is this fact that $g_{n}$ must be periodic which makes the conditions on $a$ and $b$ more restrictive than in the case when one studies (2) for $x \in \mathbf{R}$.)

To find a function $g_{n}$ which satisfies our conditions, we begin by taking $\bar{\delta}(t)$ as in Assumption 3 . Then take $g_{n}$ to be a non-zero solution of

$$
\frac{\partial_{x} g}{g}=-\frac{1}{3}\left(\frac{b}{a}-\bar{\delta}\right)+\left(\frac{1}{2}-\frac{n}{3}\right) \frac{a_{x}}{a} .
$$

Specifically, define

$$
g_{n}(x, t):=(a(x, t))^{1 / 2-n / 3} \exp \left[-\frac{1}{3} \int_{0}^{x}\left(\frac{b(y, t)}{a(y, t)}-\bar{\delta}(t)\right) d y\right]
$$

Then we have the following lemma:

Lemma 6. Take Assumptions 1, 2 and 3 as given and define $g_{n}$ as in (19). Then $g_{n}$ satisfies (C1), (C2), (C3) and (17).

Proof: Assumptions 1 and 2 imply $a$ is bounded away from zero and also bounded above, so the same is true for $(a(x, t))^{1 / 2-n / 3}$. Likewise $b$ is bounded above, as is $\bar{\delta}$, and so the exponential is bounded above and away from zero. Thus we have (C1) for an appropriate constant $k_{g}$. The regularity of $g_{n}$ can be ascertained from (19). Essentially $g_{n}$ has the same regularity as $a$ (due to the prefactor of $a^{1 / 2-n / 3}$ ) and one derivative smoother than $b$ (since $b$ appears in $(19)$ only as $\int_{0}^{x} \frac{b(y)}{a(y)} d y$ ). Thus Assumption 1 gives (C2). Observe that, for any $t \in[0, T]$ the definition of $\bar{\delta}$ and the fact that $a$ is periodic gives:

$$
\begin{aligned}
& g_{n}(M, t)=(a(M, t))^{1 / 2-n / 3} \exp {\left[-\frac{1}{3} \int_{0}^{M}\left(\frac{b(y, t)}{a(y, t)}-\bar{\delta}(t)\right) d y\right] } \\
&=(a(M, t))^{1 / 2-n / 3} \exp \left[-\frac{1}{3}(M \bar{\delta}(t)-M \bar{\delta}(t))\right] \\
&=(a(M, t))^{1 / 2-n / 3}=(a(0, t))^{1 / 2-n / 3}=g_{n}(0, t) .
\end{aligned}
$$

Thus $g_{n}$ is periodic. The same reasoning shows that its derivatives are periodic and we have (C3). Finally, rearranging the terms in (18) gives

$$
\left(\frac{3}{2}-n\right) a_{x}-b-3 a \frac{\partial_{x} g_{n}}{g_{n}}=-a \bar{\delta}(t) .
$$

Since $a>0$ and since Assumption 3 tells us that $\bar{\delta} \geq 0$, we have 17 . 
2.4. Well-posedness of (7). Now that we have selected $g_{n}$ we can prove that (7), and consequently (2), is well-posed. We will first prove uniqueness and continuous dependence of solutions, and we will then prove existence. For all of these, a uniform bound for solutions will be helpful. Lemma 6 leads to the following energy estimate:

Proposition 1. Take Assumptions 1, 2 and 3 as given and define $g_{n}$ as in (19). If $v \in L^{\infty}\left([0, T] ; H^{n}\right)$ solves (7) then

$$
\frac{d}{d t}\|v(t)\|_{H^{n}}^{2} \leq K\|v(t)\|_{H^{n}}^{2}+2(v(t), \tilde{e}(t))_{H^{n}} .
$$

Proof: Following the time-honored tradition, we compute

$$
\frac{d}{d t}\|v(t)\|_{H^{n}}^{2}=\frac{d}{d t}\|v(t)\|_{L^{2}}^{2}+\frac{d}{d t}\left\|\partial_{x}^{n} v(t)\right\|_{L^{2}}^{2}=2 \int_{X} v v_{t} d x+2 \int_{X}\left(\partial_{x}^{n} v\right) \partial_{x}^{n} v_{t} d x .
$$

Using the definition of $L_{A B C D}$, (7) can be rewritten as $v_{t}=L_{a \tilde{b} \tilde{c} \tilde{d}} v+\tilde{e}$. Thus:

$$
\begin{aligned}
\frac{d}{d t}\|v(t)\|_{H^{n}}^{2}= & 2 \int_{X} v L_{a \tilde{b} \tilde{c} \tilde{d}} v d x+2 \int_{X}\left(\partial_{x}^{n} v\right) \partial_{x}^{n}\left(L_{a \tilde{b} \tilde{c} \tilde{d}} v\right) d x \\
& +2 \int_{X} v \tilde{e} d x+2 \int_{X}\left(\partial_{x}^{n} v\right) \partial_{x}^{n} \tilde{e} d x \\
= & 2 \int_{X} v L_{a \tilde{b} \tilde{c} \tilde{d}} v d x+2 \int_{X}\left(\partial_{x}^{n} v\right) \partial_{x}^{n}\left(L_{a \tilde{b} \tilde{c} \tilde{d}} v\right) d x+2(v(t), \tilde{e}(t))_{H^{n}} .
\end{aligned}
$$

Since $n \geq 4$ and $L_{a \tilde{b} \tilde{c} \tilde{d}}$ takes three derivatives:

$$
2 \int_{X} v L_{a \tilde{b} \tilde{c} \tilde{d}} v d x \leq K\|v(t)\|_{H^{n}}^{2} .
$$

Our choice of $g_{n}$ and Lemma 6 allow us to use (16), thus:

$$
2 \int_{X} \partial_{x}^{n} v \partial_{x}^{n}\left(L_{a \tilde{b} \tilde{c} \tilde{d}} v\right) d x \leq K\|v(t)\|_{H^{n}}^{2}
$$

This proposition immediately implies:

Corollary 7. (Uniqueness and continuous dependence on initial conditions) Take Assumptions 1. 2 and 3 as given and define $g_{n}$ as in (19). Suppose that $v_{1}, v_{2} \in H_{T}^{n}$ are solutions of (7). Then for all $t \in[0, T]$,

$$
\left\|v_{1}(t)-v_{2}(t)\right\|_{H^{n}} \leq K\left\|v_{1}(0)-v_{2}(0)\right\|_{H^{n}}
$$

In particular, if $v_{1}(0)=v_{2}(0)$ then $v_{1} \equiv v_{2}$.

Proof: Let $\Delta=v_{1}-v_{2}$. Clearly

$$
\Delta_{t}=L_{a \tilde{b} \tilde{c} \tilde{d}} \Delta
$$

and $\Delta(0)=v_{1}(0)-v_{2}(0)$. Apply Proposition 1 with $\tilde{e}=0$ to get $\frac{d}{d t}\|\Delta(t)\|_{H^{n}}^{2} \leq$ $K\|\Delta(t)\|_{H^{n}}^{2}$. Gronwall's inequality then gives the estimate.

All that remains is to show that (7) does indeed have solutions. Here is our result:

Proposition 2. Take Assumptions 1, 2 and 3 as given and define $g_{n}$ as in (19). For all $v_{0} \in H^{n}$, with $n \geq 4$, there exists a function $v^{*}$ with the following properties:

- $v^{*} \in L^{\infty}\left([0, T] ; H^{n}\right)$ with $\|v\|_{H_{T}^{n}} \leq K\left(\left\|v_{0}\right\|_{H^{n}}+1\right)$, 
- for all $0 \leq n^{\prime}<n, v^{*} \in C\left([0, T] ; H^{n^{\prime}}\right)$ with $\|v\|_{H_{T}^{n^{\prime}}} \leq K\left(\left\|v_{0}\right\|_{H^{n}}+1\right)$,

- $v^{*}(x, 0)=v_{0}$ and

- $v^{*}(x, t)$ solves (7).

Remark 6. This proposition, Corollary 7, and Lemma 4 imply the main result, Theorem 1 .

Proof: (Proposition 2) We first regularize (7). Let $\mathcal{J}_{\epsilon} \psi:=\eta_{\epsilon} * \psi$ where $*$ is the periodic convolution and $\eta_{\epsilon}$ is "Evans' standard mollifier" 9]. For utility we record the following properties of $\mathcal{J}_{\epsilon}$ :

Theorem 8. (1) For all $\epsilon>0, s_{1}, s_{2} \in \mathbf{R},\left\|\mathcal{J}_{\epsilon}\right\|_{H^{s_{1}} \rightarrow H^{s_{2}}} \leq C\left(\epsilon, s_{1}, s_{2}\right)$.

(2) If $\phi \in H^{s}$, then $\lim _{\epsilon \rightarrow 0^{+}}\left\|\mathcal{J}_{\epsilon} \phi-\phi\right\|_{H^{s}}=0$.

(3) For all $\epsilon>0, s \in \mathbf{R},\left\|\mathcal{J}_{\epsilon}\right\|_{H^{s} \rightarrow H^{s}} \leq C(s)$. Specifically, $C(s)$ does not depend on $\epsilon$.

(4) For all $\epsilon>0, s \in \mathbf{R}$, and $\phi, \psi \in H^{s},\left(\mathcal{J}_{\epsilon} \phi, \psi\right)_{H^{s}}=\left(\phi, \mathcal{J}_{\epsilon} \psi\right)_{H^{s}}$.

(5) $\mathcal{J}_{\epsilon} \partial_{x}=\partial_{x} \mathcal{J}_{\epsilon}$.

Consider now the following regularized initial vale problem:

$$
v_{t}^{\epsilon}=\mathcal{J}_{\epsilon}\left(a \mathcal{J}_{\epsilon} v_{x x x}^{\epsilon}+\tilde{b} \mathcal{J}_{\epsilon} v_{x x}^{\epsilon}+\tilde{c} \mathcal{J}_{\epsilon} v_{x}^{\epsilon}+\tilde{d} \mathcal{J}_{\epsilon} v^{\epsilon}+\tilde{e}\right)
$$

with

$$
v^{\epsilon}(x, 0)=v_{0} \in H^{n} .
$$

Due to the presence of the mollifiers, the right hand side of (21) defines a Lipschitz map from $H^{n}$ to itself. Since $\mathcal{J}_{\epsilon}$ commutes with derivatives, we can rewrite the right hand side of (21) as:

$$
\mathcal{J}_{\epsilon} L_{a \tilde{b} \tilde{c} \tilde{d}} \mathcal{J}_{\epsilon} v^{\epsilon}+\mathcal{J}_{\epsilon} \tilde{e}
$$

So we have from the standard existence theory for ODEs on a Banach spaces:

Lemma 9. For all $\epsilon>0$ there exist $T_{\epsilon}>0$ and

$$
v^{\epsilon}(x, t) \in C^{1}\left(\left[0, T_{\epsilon}\right] ; H^{n}\right)
$$

which solves 21) and 22.

Here, $T_{\epsilon}>0$ depends, possibly badly, on $\epsilon$. We now prove an analog of Proposition 1 for solutions of (21). Specifically, we have, just as in the proof of Proposition 1 .

$$
\begin{aligned}
\frac{d}{d t}\left\|v^{\epsilon}(t)\right\|_{H^{n}}^{2}=2 \int_{X} v^{\epsilon} \mathcal{J}_{\epsilon} & \left(L_{a \tilde{b} \tilde{c} \tilde{d}} \mathcal{J}_{\epsilon} v^{\epsilon}\right) d x \\
& +2 \int_{X} \partial_{x}^{n} v^{\epsilon} \partial_{x}^{n} \mathcal{J}_{\epsilon}\left(L_{a \tilde{b} \tilde{c} \tilde{d}} \mathcal{J}_{\epsilon} v^{\epsilon}\right) d x+2\left(v^{\epsilon}(t), \mathcal{J}_{\epsilon} \tilde{e}(t)\right)_{H^{n}} .
\end{aligned}
$$

Using the fact that $\mathcal{J}_{\epsilon}$ is self-adjoint (Theorem 8 , part (4)) gives:

$$
\begin{aligned}
\frac{d}{d t}\left\|v^{\epsilon}(t)\right\|_{H^{n}}^{2}=2 \int_{X} & \mathcal{J}_{\epsilon} v^{\epsilon}\left(L_{a \tilde{b} \tilde{c} \tilde{d}} \mathcal{J}_{\epsilon} v^{\epsilon}\right) d x \\
& +2 \int_{X} \partial_{x}^{n} \mathcal{J}_{\epsilon} v^{\epsilon} \partial_{x}^{n}\left(L_{a \tilde{b} \tilde{c} \tilde{d}} \mathcal{J}_{\epsilon} v^{\epsilon}\right) d x+2\left(\mathcal{J}_{\epsilon} v^{\epsilon}(t), \tilde{e}(t)\right)_{H^{n}}
\end{aligned}
$$


The first term is easily bounded by $K\left\|\mathcal{J}_{\epsilon} v^{\epsilon}\right\|_{H^{n}}^{2}$, since $n \geq 4$. We can apply Lemma 5 and the estimate (16) to the second term, with $\phi=\mathcal{J}_{\epsilon} v^{\epsilon}$. (The mollifiers were arranged precisely in (21) so that we could do this.) We then have:

$$
\frac{d}{d t}\left\|v^{\epsilon}(t)\right\|_{H^{n}}^{2} \leq K\left\|\mathcal{J}_{\epsilon} v^{\epsilon}(t)\right\|_{H^{n}}^{2}+2\left(\mathcal{J}_{\epsilon} v^{\epsilon}(t), \tilde{e}(t)\right)_{H^{n}} .
$$

Note that the constant $K$ here (and any such constants which appear below) do not depend on $\epsilon$.

Since $\left\|\mathcal{J}_{\epsilon} \phi\right\|_{H^{n}} \leq C(n)\|\phi\|_{H^{n}}$ where $C(n)$ does not depend on $\epsilon$, we have, with the Cauchy-Schwarz inequality:

$$
\frac{d}{d t}\left\|v^{\epsilon}(t)\right\|_{H^{n}}^{2} \leq K\left(\left\|v^{\epsilon}(t)\right\|_{H^{n}}^{2}+\left\|v^{\epsilon}(t)\right\|_{H^{n}}\right) \leq K\left(1+\left\|v^{\epsilon}(t)\right\|_{H^{n}}^{2}\right) .
$$

Applying Gronwall's inequality to this gives, for all $t \in\left[0, T_{\epsilon}\right]$.

$$
\left\|v^{\epsilon}(t)\right\|_{H^{n}}^{2} \leq\left(1+\left\|v_{0}\right\|_{H^{n}}^{2}\right) e^{K t}-1 \leq K\left(\left\|v_{0}\right\|_{H^{n}}^{2}+1\right) .
$$

Thus we have $\left\|v^{\epsilon}\left(T_{\epsilon}\right)\right\|_{H^{n}}<\infty$. This means that we can continue the solution; we can do so indefinitely given the form of (24). We conclude that, for all $\epsilon$,

$$
v^{\epsilon} \in C^{1}\left([0, T] ; H^{n}\right) \text {. }
$$

Which is to say that the solutions $v^{\epsilon}$ all exist for a common time interval.

Since $n \geq 4$, we have $H^{n} \subset C^{3}$. Thus the estimate (24) implies that

$$
\sup _{t \in[0, T]}\left\|v^{\epsilon}(t)\right\|_{C^{3}} \leq K\left(\left\|v_{0}\right\|_{H^{n}}+1\right) .
$$

And since $v^{\epsilon}$ solves 21], we have

$$
\sup _{t \in[0, T]}\left\|v_{t}^{\epsilon}(t)\right\|_{C} \leq K \sup _{t \in[0, T]}\|v(t)\|_{C^{3}} \leq K\left(\left\|v_{0}\right\|_{H^{n}}+1\right) .
$$

In particular, there is a constant $K$ independent of $\epsilon$ :

$$
\sup _{t \in[0, T]}\left(\left\|v_{x}^{\epsilon}\right\|_{C}+\left\|v_{t}^{\epsilon}\right\|_{C}\right) \leq K\left(\left\|v_{0}\right\|_{H^{n}}+1\right)
$$

This implies that $\left\{v^{\epsilon}\right\}_{\epsilon>0}$ is a uniformly bounded and equicontinuous family of functions defined for $(x, t) \in[0, M] \times[0, T] . \quad[0, M] \times[0, T] \in \mathbf{R}^{2}$ is compact, and so we apply the Arzela-Ascoli theorem to conclude that there is a function $v^{*} \in C([0, M] \times[0, T])$ and subsequence $\left\{\epsilon_{j}\right\}$ (with $\left.\lim _{j \rightarrow \infty} \epsilon_{j}=0\right)$ such that

$$
\lim _{j \rightarrow \infty}\left\|v^{\epsilon_{j}}-v^{*}\right\|_{C([0, M] \times[0, T])}=\lim _{j \rightarrow \infty}\left\|v^{\epsilon_{j}}-v^{*}\right\|_{L_{T}^{\infty}}=0 .
$$

We have from interpolation, for all $n^{\prime} \in[0, n)$ :

$$
\left\|v^{\epsilon_{j}}(t)-v^{\epsilon_{j^{\prime}}}(t)\right\|_{H^{n^{\prime}}} \leq\left\|v^{\epsilon_{j}}(t)-v^{\epsilon_{j^{\prime}}}(t)\right\|_{L^{2}}^{q}\left\|v^{\epsilon_{j}}(t)-v^{\epsilon_{j^{\prime}}}(t)\right\|_{H^{n}}^{r}
$$

where $q=1-n^{\prime} / n$ and $r=n^{\prime} / n$. The estimate 24) implies that:

$$
\left\|v^{\epsilon_{j}}(t)-v^{\epsilon_{j^{\prime}}}(t)\right\|_{H^{n^{\prime}}} \leq K\left(\left\|v_{0}\right\|_{H^{n}}+1\right)^{r}\left\|v^{\epsilon_{j}}(t)-v^{\epsilon_{j^{\prime}}}(t)\right\|_{L^{2}}^{q} .
$$

Since $[0, M]$ is compact, $L^{\infty} \subset L^{2}$ :

$$
\left\|v^{\epsilon_{j}}-v^{\epsilon_{j^{\prime}}}\right\|_{H_{T}^{n^{\prime}}} \leq K\left(\left\|v_{0}\right\|_{H^{n}}+1\right)^{r}\left\|v^{\epsilon_{j}}-v^{\epsilon_{j^{\prime}}}\right\|_{L_{T}^{\infty}}^{q} .
$$

Of course, since $v^{\epsilon_{j}} \rightarrow v^{*}$ uniformly, $\left\{v^{\epsilon_{j}}\right\}$ is a Cauchy sequence in the $C\left([0, T] ; L^{\infty}\right)$ topology. Moreover, we know that for all $\epsilon, v^{\epsilon} \in C^{1}\left([0, T] ; H^{n^{\prime}}\right)$ by 25). Thus 27] 
implies $\left\{v^{\epsilon_{j}}\right\}$ is a Cauchy sequence in the $C\left([0, T] ; H^{n^{\prime}}\right)$ topology, and therefore convergent. Of course, the limit must be $v^{*}$. And so, for all $n \in\left[0, n^{\prime}\right)$ :

$$
v^{*} \in C\left([0, T] ; H^{n^{\prime}}\right) .
$$

Moreover, since the $v^{\epsilon}$ are in $C\left([0, T] ; H^{n}\right)$, for each $t \in[0, T]$ we have $\left\{v^{\epsilon_{j}}(t)\right\}$ a bounded sequence in $H^{n}$. Thus this sequence has a weak limit in $H^{n}$. Call the limit $v^{* *}(t)$. We have $v^{* *} \in L^{\infty}\left([0, T] ; H^{n}\right)$. An elementary argument using the fact that limits are unique shows that $v^{* *}=v^{*}$. But this then means

$$
v^{*} \in L^{\infty}\left([0, T] ; H^{n}\right) .
$$

We claim that $v^{*}$ solves (7) with initial condition 22 . For all $\epsilon$, we have

$$
v^{\epsilon}(x, t)=v_{0}(x)+\int_{0}^{t}\left(\mathcal{J}_{\epsilon} L_{a \tilde{b} \tilde{c} \tilde{d}} \mathcal{J}_{\epsilon} v^{\epsilon}(x, s)+\mathcal{J}_{\epsilon} \tilde{e}(s)\right) d s .
$$

Recall that $L_{a \tilde{b} \tilde{c} \tilde{d}}$ takes three derivatives and $\lim _{\epsilon \rightarrow 0^{+}}\left\|\mathcal{J}_{\epsilon} \phi-\phi\right\|_{H^{s}}=0$ for any $\phi \in H^{s}$. Moreover $v^{\epsilon_{j}} \rightarrow v^{*}$ as $j \rightarrow \infty$ in $C\left([0, T] ; H^{n^{\prime}}\right)$ for any $n^{\prime} \in[0, n)$ with $n \geq 4$. And so we have no difficulty passing to the limit in 28 along the subsequence $\left\{\epsilon_{j}\right\}$ to get

$$
v^{*}(x, t)=v_{0}(x)+\int_{0}^{t}\left(L_{a \tilde{b} \tilde{c} \tilde{d}} v^{*}(x, s)+\tilde{e}(s)\right) d s .
$$

This implies that $v^{*}$ sastifies (7) and (22).

We now prove the extensions.

Proof: (Theorem 2) Suppose that

$$
\bar{\delta}(t) \geq \delta_{0}>0 .
$$

Follow the proof of Theorem 2 until 23]. Then 24] implies

$$
\frac{d}{d t}\left\|v^{\epsilon}(t)\right\|_{H^{n}}^{2} \leq 2 \int_{X} \partial_{x}^{n} \mathcal{J}_{\epsilon} v^{\epsilon} \partial_{x}^{n}\left(L_{a \tilde{b} \tilde{c} \tilde{d}} \mathcal{J}_{\epsilon} v^{\epsilon}\right) d x+K\left(\left\|v_{0}\right\|_{H^{n}}^{2}+1\right)
$$

which, upon subtracting from both sides, gives:

$$
\begin{aligned}
\frac{d}{d t}\left\|v^{\epsilon}(t)\right\|_{H^{n}}^{2}-2 \int_{X} & {\left[\left(\frac{3}{2}-n\right) a_{x}-\tilde{b}\right]\left[\partial_{x}^{n+1} \mathcal{J}_{\epsilon} v^{\epsilon}\right]^{2} } \\
& \leq 2 \int_{X} \partial_{x}^{n} \mathcal{J}_{\epsilon} v^{\epsilon} \partial_{x}^{n}\left(L_{a \tilde{b} \tilde{c} \tilde{d}} \mathcal{J}_{\epsilon} v^{\epsilon}\right) d x \\
& -2 \int_{X}\left[\left(\frac{3}{2}-n\right) a_{x}-\tilde{b}\right]\left[\partial_{x}^{n+1} \mathcal{J}_{\epsilon} v^{\epsilon}\right]^{2}+K\left(\left\|v_{0}\right\|_{H^{n}}^{2}+1\right) .
\end{aligned}
$$

Applying (9) on the right hand side we get:

$$
\frac{d}{d t}\left\|v^{\epsilon}(t)\right\|_{H^{n}}^{2}-2 \int_{X}\left[\left(\frac{3}{2}-n\right) a_{x}-\tilde{b}\right]\left[\partial_{x}^{n+1} \mathcal{J}_{\epsilon} v^{\epsilon}\right]^{2} \leq K\left(\left\|v_{0}\right\|_{H^{n}}^{2}+1\right) .
$$

Our choice 19$]$ for $g_{n}$ and 20$]$ give:

$$
\frac{d}{d t}\left\|v^{\epsilon}(t)\right\|_{H^{n}}^{2}+2 \bar{\delta}(t) \int_{X} a\left[\partial_{x}^{n+1} \mathcal{J}_{\epsilon} v^{\epsilon}\right]^{2} \leq K\left(\left\|v_{0}\right\|_{H^{n}}^{2}+1\right) .
$$

Integrating both sides with respect to time gives us the following estimate:

$$
\left\|v^{\epsilon}(t)\right\|_{H^{n}}^{2}+2 \int_{0}^{t} \bar{\delta}(s) \int_{X} a(s)\left[\partial_{x}^{n+1} \mathcal{J}_{\epsilon} v^{\epsilon}(s)\right]^{2} d s \leq K\left(\left\|v_{0}\right\|_{H^{n}}^{2}+1\right) .
$$


Thus we have, for all $t \in[0, T]$ :

$$
\int_{0}^{t} \bar{\delta}(s) \int_{X} a(s)\left[\partial_{x}^{n+1} \mathcal{J}_{\epsilon} v^{\epsilon}(s)\right]^{2} d s \leq K\left(\left\|v_{0}\right\|_{H^{n}}^{2}+1\right) .
$$

Assumption 2 and 2 29) imply that

$$
\left.\left.\int_{0}^{t} \| \partial_{x}^{n+1} \mathcal{J}_{\epsilon} v^{\epsilon}(s)\right) \|_{L^{2}}^{2} d s \leq \frac{1}{\delta_{0} a_{0}} \int_{0}^{t} \bar{\delta}(s) \int_{X} a(s)\left[\partial_{x}^{n+1} \mathcal{J}_{\epsilon} v^{\epsilon}(s)\right)\right]^{2} d s .
$$

Thus, for any $\epsilon>0$ :

$$
\left.\int_{0}^{T} \| \partial_{x}^{n+1} \mathcal{J}_{\epsilon} v^{\epsilon}(s)\right) \|_{L^{2}}^{2} d s \leq K\left(\left\|v_{0}\right\|_{H^{n}}^{2}+1\right)
$$

And so $\left\{\mathcal{J}_{\epsilon} v^{\epsilon}\right\}$ is a bounded set in $L^{2}\left([0, T] ; H^{n+1}\right)$. It then has a weakly convergent subsequence, whose limit we call $v^{* * *} \in L^{2}\left([0, T] ; H^{n+1}\right)$. Given that $v_{\epsilon} \rightarrow v^{*}$ strongly in $C\left([0, T] ; H^{n^{\prime}}\right)$ when $0 \leq n^{\prime}<n$, it is straightforward to conclude that $\mathcal{J}_{\epsilon} v^{\epsilon} \rightarrow v^{*}$ strongly in $L^{2}\left([0, T] ; H^{n^{\prime}}\right)$. Uniqueness of limits thus implies $v^{*}=v^{* * *}$. Thus $v^{*} \in L^{2}\left([0, T] ; H^{n+1}\right)$. As norms are lower semi-continuous with respect to weak limits we have

$$
\left.\int_{0}^{T}\left\|v^{*}(s)\right\|_{H^{n+1}}^{2} d s \leq \lim _{\epsilon \rightarrow 0} \int_{0}^{T} \| \mathcal{J}_{\epsilon} v^{\epsilon}(s)\right) \|_{H^{n+1}}^{2} d s \leq K\left(\left\|v_{0}\right\|_{H^{n}}^{2}+1\right) .
$$

All that remains is to show that $v^{*} \in C\left([0, T] ; H^{n}\right)$. Since $v^{*} \in L^{2}\left([0, T] ; H^{n+1}\right)$, we see that for a.e. $t \in[0, T], v^{*}(t) \in H^{n+1}$. Let $\tau>0$ be such a time. Then we can rerun the existence argument in its entirety using $v^{*}(\tau) \in H^{n+1}$ as the initial condition, but in the smoother space (hence the additional regularity requirements in the statement of Theorem 2). Call this new solution $v^{\star}$. By Proposition 2, we have $v^{\star} \in C\left([\tau, T] ; H^{n^{\prime}+1}\right)$ for all $n^{\prime} \in[0, n)$. By uniqueness, it must be the case that $v^{\star}=v^{*}$ for $t \in[\tau, T]$. Lastly, we can conclude that

$$
\lim _{t \rightarrow 0^{+}}\left\|v^{*}-v_{0}\right\|_{H^{n}}=0
$$

the details of this step are the same as in the proof of Theorem 18 below. Therefore, we get $v^{*} \in C\left([0, T] ; H^{n}\right)$, and since $\tau$ can be taken arbitrarily close to 0 , we also have $v^{*} \in C\left((0, T] ; H^{n^{\prime}+1}\right)$ as claimed.

Proof: (Theorem 3) Suppose that

$$
\bar{\delta}(t)=\frac{1}{M} \int_{0}^{M} \frac{b(y, t)}{|a(y, t)|} d y=0 .
$$

The crux of the argument here is that in this case (2) is, for all intents and purposes, reversible. Specifically, suppose that $u(x, t)$ solves (2) with initial conditions $u_{0}(x)$. Then let $w(x, t)=u\left(M-x, t_{0}-t\right)$ where $t_{0} \in(0, T]$. Then

$$
w_{t}=\bar{a} w_{x x x}-\bar{b} w_{x x}+\bar{c} w_{x}-\bar{d} w+\bar{e}
$$

where $\bar{a}(x, t)=a\left(M-x, t_{0}-t\right), \bar{b}(x, t)=b\left(M-x, t_{0}-t\right)$ and so on. Solving (2) for $t$ backwards from $t_{0}$ is therefore equivalent to solving (31) forward in time. The claim is that (31) meets all the Assumptions, and is thus solvable. Clearly the coefficients on the right hand side of (31) satisfy the regularity requirements 
of Assumption 1. Likewise it is clear that $\inf _{x \in X}|\bar{a}(x, t)|=\inf _{x \in X}|a(x, t)|$ and so Assumption 2 is also met. Lastly,

$$
\frac{1}{M} \int_{0}^{M} \frac{\bar{b}(y, t)}{|\bar{a}(y, t)|} d y=\frac{1}{M} \int_{0}^{M} \frac{b(M-y, t)}{|a(M-y, t)|} d y=\frac{1}{M} \int_{0}^{M} \frac{b(y, t)}{|a(y, t)|} d y=0 .
$$

Thus Assumption 3 is met, and so we apply Theorem 1 to get solutions of (31). In combination with the energy estimates we have, the reversibility implies

$$
u \in C\left([0, T] ; H^{n}\right) ;
$$

we omit the argument at present, as it is essentially the same as the argument in the proof of Theorem 18 below.

2.5. Ill-posedness when $\bar{\delta}<0$. Let us define $L$ as:

$$
L u:=a(x) u_{x x x}+b(x) u_{x x}+c(x) u_{x}+d(x) u .
$$

We have:

Theorem 10. Suppos $\S^{1}$ that $a, b, c$ and $d$ are in $C^{\infty}(X)$. If

$$
\inf _{x \in X} a(x)=a_{0}>0 \quad \text { and } \quad \bar{\delta}=\frac{1}{M} \int_{0}^{M} \frac{b(y)}{a(y)} d y \neq 0
$$

then there is a sequence of eigenvalues $\left\{\lambda_{j}\right\}_{j \in \mathbf{N}}$ of L for which

$$
\lim _{j \rightarrow \infty}-\bar{\delta} \Re\left(\lambda_{j}\right)=+\infty .
$$

Notice that if $\bar{\delta}<0$ then this proposition implies that $L$ has eigenvalues with arbitrarily large real part. And thus $u_{t}=L u$ is ill-posed.

Proof: We treat the special case when

$$
L u=u_{x x x}+\bar{\delta} u_{x x}+c(x) u_{x}+d(x) u .
$$

The general case follows by a change of variables, which we postpone for the moment. This portion of the proof is similar to the proof that uniformly elliptic operators have eigenvalues which tend to infinity in 9 . Note that we are viewing $L$ as an unbounded linear operator from $L^{2}$ to itself whose domain is $H^{3}$.

First we claim that $L$ has a sequence of eigenvalues $\left\{\lambda_{j}\right\}_{j \in \mathbf{N}}$ for which

$$
\lim _{j \rightarrow \infty}\left|\lambda_{j}\right|=\infty .
$$

Since we are working on the periodic interval, $L$ is a relatively compact perturbation of $\partial_{x}^{3}$. Thus we have $\sigma_{\text {ess }}(L)=\sigma_{\text {ess }}\left(\partial_{x}^{3}\right)=\{\}$. This implies that the resolvent set of $L$ is nonempty. Let $\omega$ be a point in the resolvent. Let $K=(L-\omega)^{-1}$. We have $K$ is bounded linear map from $L^{2}$ to $H^{3}$. The Rellich-Kondrachov theorem implies that $H^{3}$ is compactly embedded in $L^{2}$, and thus we conclude $K$ is a compact map from $L^{2}$ to itself. This implies that $K$ has a sequence of non-zero eigenvalues $\left\{\mu_{j}\right\}_{j \in \mathbf{N}}$ for which $\lim _{j \rightarrow \infty} \mu_{j}=0$. Since $K$ is bounded from $L^{2}$ to $H^{3}$ we have functions $u_{j} \in H^{3}$ so that $K u_{j}=\mu_{j} u_{j}$. Since $K=(L-\omega)^{-1}$ a short calculation shows

$$
L u_{j}=\left(\omega+\frac{1}{\mu_{j}}\right) u_{j} .
$$

\footnotetext{
${ }^{1}$ We take smooth periodic coefficients here for simplicity.
} 
Thus $\lambda_{j}=\omega+\frac{1}{\mu_{j}}$ is an eigenvalue of $L$. Since the $\mu_{j}$ tend to zero, the $\left|\lambda_{j}\right|$ tend to infinity. Thus the claim is shown.

Next, let $u$ and $\lambda$ be an eigenfunction/eigenvalue pair for $L$, with $\|u\|_{L^{2}}=1$. Since $L u=\lambda u$ we have

$$
\lambda=\int_{X} L u(x) \bar{u}(x) d x=\int_{X}\left(u_{x x x}+\bar{\delta} u_{x x}+c(x) u_{x}+d(x) u\right) \bar{u} d x .
$$

Now, by integration by parts, $\int_{X} u_{x x x} \bar{u} d x=-\int_{X} u \bar{u}_{x x x} d x$. This implies that $\Re \int_{X} u_{x x x} \bar{u} d x=0$. Likewise, integration by parts shows $\int_{X} \bar{\delta} u_{x x} \bar{u} d x=-\bar{\delta}\left\|u_{x}\right\|_{L^{2}}^{2}$. And so taking the real part of 32 gives:

$$
\Re(\lambda)=-\bar{\delta}\left\|u_{x}\right\|_{L^{2}}^{2}+\Gamma
$$

where

$$
\Gamma:=\Re\left[\int_{X}\left(c(x) u_{x}+d(x) u\right) \bar{u} d x\right] .
$$

A straightforward calculation shows

$$
|\Gamma| \leq K\|u\|_{L^{2}}^{2}=K
$$

where $K>0$ depends only on $c$ and $d$. Notice that (33) implies, when $\bar{\delta}<0$ that $\Re(\lambda) \geq-K$ for some constant $K$ which depends only $c$ and $d$. On the other hand if $\bar{\delta}>0$ then we have $\Re(\lambda) \leq K$. Which is to say

$$
\bar{\delta} \Re(\lambda) \leq|\bar{\delta}| K .
$$

for all eigenvalues $\lambda$. Solving (33) for $\left\|u_{x}\right\|_{L^{2}}$ gives:

$$
\left\|u_{x}\right\|_{L^{2}}^{2}=-\bar{\delta}^{-1}(\Re(\lambda)-\Gamma) \leq k_{1}|\Re(\lambda)|+k_{2}
$$

for positive constants $k_{1}$ and $k_{2}$ which do not depend on $\lambda$.

Taking imaginary parts of 32 gives:

$$
\Im(\lambda)=\int_{X} u_{x x x} \bar{u} d x+\Omega
$$

where

$$
\Omega=\Im\left[\int_{X}\left(c(x) u_{x}+d(x) u\right) \bar{u} d x\right] .
$$

One can show that using the Cauchy-Schwarz inequality that:

$$
\Omega \leq K\left\|u_{x}\right\|_{L^{2}}\|u\|_{L^{2}}=K\left\|u_{x}\right\|_{L^{2}} .
$$

where $K$ depends only on $c$.

An integration by parts and Cauchy-Schwarz give

$$
\int_{X} u_{x x x} \bar{u} d x=-\int_{X} u_{x x} \bar{u}_{x} d x \leq\left\|u_{x x}\right\|_{L^{2}}\left\|u_{x}\right\|_{L^{2}} .
$$

So we have

$$
|\Im(\lambda)| \leq\left(\left\|u_{x x}\right\|_{L^{2}}+K\right)\left\|u_{x}\right\|_{L^{2}} .
$$

Using (35) gives:

$$
|\Im(\lambda)| \leq\left(\left\|u_{x x}\right\|_{L^{2}}+K\right) \sqrt{k_{1}|\Re(\lambda)|+k_{2}} .
$$


Now, since $L u=\lambda u$ we have

$$
\lambda \int_{X} u \bar{u}_{x x} d x=\int_{X}\left(u_{x x x}+\bar{\delta} u_{x x}+c(x) u_{x}+d(x) u\right) \bar{u}_{x x} d x .
$$

An integration by parts shows that the left hand side equals $-\lambda\left\|u_{x}\right\|_{L^{2}}^{2}$. Therefore we have, after a rearrangement of terms:

$$
\bar{\delta}\left\|u_{x x}\right\|_{L^{2}}^{2}=-\lambda\left\|u_{x}\right\|_{L^{2}}^{2}-\int_{X}\left(u_{x x x}+c(x) u_{x}+d(x) u\right) \bar{u}_{x x} d x .
$$

Integration by parts shows that $\Re \int_{X} u_{x x x} \bar{u}_{x x} d x=0$. Taking the real part of the last equation then gives

$$
\bar{\delta}\left\|u_{x x}\right\|_{L^{2}}^{2}=-\Re(\lambda)\left\|u_{x}\right\|_{L^{2}}^{2}+\Theta
$$

with

$$
\Theta:=-\Re\left[\int_{X}\left(c(x) u_{x}+d(x) u\right) \bar{u}_{x x} d x\right] .
$$

Integration by parts shows that

$$
|\Theta| \leq K\left(\left\|u_{x}\right\|_{L^{2}}^{2}+1\right)
$$

where $K>0$ depends on $c$ and $d$.

$$
\left\|u_{x x}\right\|_{L^{2}}^{2}=-\bar{\delta}^{-1} \Re(\lambda)\left\|u_{x}\right\|_{L^{2}}^{2}+\bar{\delta}^{-1} \Theta
$$

and using the estimate on $\Theta$ and also $(35)$ we get:

$$
\left\|u_{x x}\right\|_{L^{2}}^{2} \leq k_{3} \Re(\lambda)^{2}+k_{4}
$$

for positive constants $k_{3}$ and $k_{4}$ which do not depend on $\lambda$. Inserting this into (36) gives

$$
|\Im(\lambda)| \leq\left(\sqrt{k_{3} \Re(\lambda)^{2}+k_{4}}+K\right) \sqrt{k_{1}|\Re(\lambda)|+k_{2}} .
$$

Now suppose that $\bar{\delta}<0$. Then (34) tells us that $\Re(\lambda) \geq-K<0$. This, with (38) implies that all the eigenvalues of $L$ are in the set

$$
\Sigma:=\left\{z=x+i y \in \mathbf{C}: x>-K \text { and }|y| \leq\left(\sqrt{k_{3} x^{2}+k_{4}}+K\right) \sqrt{k_{1}|x|+k_{2}} .\right\}
$$

But we also know that there is a sequence of eigenvalues of $L$ for which $|\lambda| \rightarrow \infty$. A sequence which diverges to infinity with $\Sigma$ must have $\Re(\lambda) \rightarrow+\infty$. The situation when $\bar{\delta}>0$ is similar.

Thus we have shown our result for the case when $a=1$ and $b(x)=\bar{\delta}$. A change of variables much like the one we used in the study of 2 will allow us to reduce the general case to this one, though there is one wrinkle. First set

$$
\xi(x)=\int_{0}^{x} a^{-1 / 3}(y) d y .
$$

so that $d \xi / d x=a^{-1 / 3}(x)$. By assumption that $a \geq a_{0}$ we see that $\xi$ is a strictly increasing function, and thus invertible. Then define $\tilde{v}(\eta)$ by $u(x)=\tilde{v}(\xi(x))$. A tedious but routine calculation shows that

$$
L u=a(x)\left(\xi^{\prime}\right)^{3} \tilde{v}_{\eta \eta \eta}+\left(3 a(x) \xi^{\prime} \xi^{\prime \prime}+b(x)\left(\xi^{\prime}\right)^{2}\right) \tilde{v}_{\eta \eta}+c_{1}(\eta) \tilde{v}_{\eta}+d_{1}(\eta) \tilde{v} .
$$

Formulae for the functions $c_{1}$ and $d_{1}$ are omitted, as they are complicated and not useful. 
Our choice for $\xi$ was made so that $a(x)\left(\xi^{\prime}\right)^{3}=1$. Likewise

$$
\left(3 a(x) \xi^{\prime} \xi^{\prime \prime}+b(x)\left(\xi^{\prime}\right)^{2}\right)=a^{-2 / 3}(x)\left(b(x)-a^{\prime}(x)\right)=: B(\eta) .
$$

Observe that

$$
\int_{0}^{\tilde{M}} B(\eta) d \eta=\int_{0}^{M} B(\xi(x)) \xi^{\prime}(x) d x .
$$

Here $\tilde{M}=\xi^{-1}(M)$. Since $\xi$ is increasing, $\tilde{M}>0$. And thus

$$
\begin{aligned}
\int_{0}^{\tilde{M}} B(\eta) d \eta=\int_{0}^{M} a^{-2 / 3}(x)\left(b(x)-a^{\prime}(x)\right) & a^{-1 / 3}(x) d x \\
& =\int_{0}^{M}\left(\frac{b(x)}{a(x)}-\frac{a^{\prime}(x)}{a(x)}\right) d x=M \bar{\delta} .
\end{aligned}
$$

For this reason, set $\bar{B}=\frac{M}{\tilde{M}} \bar{\delta} . \bar{B}$ has the same sign as $\bar{\delta}$. Define

$$
\tilde{L} \tilde{v}:=\tilde{v}_{\eta \eta \eta}+B(\eta) \tilde{v}_{\eta \eta}+c_{1}(\eta) \tilde{v}_{\eta}+d_{1}(\eta) \tilde{v} .
$$

Since $\tilde{L}$ is obtained from $L$ by a change of variables, clearly they have the same eigenvalues. Following (19), let $\tilde{v}=g v$ where

$$
g=\exp \left(-\frac{1}{3} \int_{0}^{x}(B(s)-\bar{B}) d s\right) .
$$

A quick calculation shows that if $\tilde{L} \tilde{v}=\lambda \tilde{v}$ then

$$
v_{\eta \eta \eta}+\frac{M}{\tilde{M}} \bar{\delta} v_{\eta \eta}+c_{2}(\eta) v_{\eta}+d_{2}(\eta) v=\lambda v
$$

where $c_{2}$ and $d_{2}$ are functions whose formulae we omit. The operator on the left hand side is exactly of the form we first studied in the proof. Since $\frac{M}{\tilde{M}} \bar{\delta}$ has the same sign as $\bar{\delta}$, we are done.

\section{WELL-POSEDNESS OF THE $K(2,2)$ IVP}

Recall that the $K(2,2)$ equation (1) is:

$$
u_{t}=2 u u_{x x x}+6 u_{x} u_{x x}+2 u u_{x} .
$$

In this section we prove that this is well-posed for for uniformly positive $H^{n}$ initial data on the periodic interval $X$. The method mirrors that which worked for (2). Specifically, we change variables and then prove energy estimates. For the linear equation (2) the change of variables was linear, and was given by (6) and (19). It turns out that applying (6) and (19) to a solution $u$ of (1) does in fact lead to a new formulation for (1) in which one can prove a priori estimates on the $H^{n}$ norm. This is done as follows. Formally, (1) is an equation of the form (2) with $a=2 u$, $b=6 u_{x}$ and $c=2 u$. Inserting these choices for $a$ and $b$ into $\sqrt{19}$ yields:

$$
g_{n}(x, t):=(2 u(x, t))^{1 / 2-n / 3} \exp \left[-\frac{1}{3} \int_{0}^{x}\left(\frac{6 u_{x}(y, t)}{2 u(y, t)}-\bar{\delta}(t)\right) d y\right]
$$


where $\bar{\delta}(t)=\frac{1}{M} \int_{0}^{M} \frac{6 u_{x}(y, t)}{2 u(y, t)} d y$, as in Assumption 3. Now, $u_{x} / u=\partial_{x}(\ln (u))$ and we are assuming $u$ is periodic and bounded away from zero. Thus we have $\bar{\delta}(t)=0$. With this, we can evaluate $g_{n}$ above to get:

$$
g_{n}(x, t)=C(t) u^{-1 / 2-n / 3}
$$

for a function $C(t)$ which does not depend on $x$. For simplicity we take $C(t)=1$. Following (6), we set $w=u / g_{n}$. All together, this suggests that changing variables in (1) via

$$
w(x, t):=[u(x, t)]^{\frac{9+2 n}{6}}
$$

will lead to estimates on the $H^{n}$ norm of $w$. And so we set

$$
\beta:=\frac{6}{9+2 n}
$$

and define $w$ as

$$
u=w^{\beta} .
$$

Note that for $n \geq 0, \beta \in(0,1)$. This nonlinear change of variables is completely modified in proving existence of solutions. It is worth remarking that while the linear estimates provided a roadmap for finding this change of variables, our nonlinear results do not rely on the results from the previous section.

The definition of $w$ implies:

$$
\begin{gathered}
u_{x}=\beta w^{\beta-1} w_{x}, \quad u_{x x}=\beta(\beta-1) w^{\beta-2} w_{x}^{2}+\beta w^{\beta-1} w_{x x}, \\
u_{x x x}=\beta(\beta-1)(\beta-2) w^{\beta-3} w_{x}^{3}+3 \beta(\beta-1) w^{\beta-2} w_{x} w_{x x}+\beta w^{\beta-1} w_{x x x} .
\end{gathered}
$$

Since $\beta \in(0,1)$, there are negative powers of $u$ in the above formulae. Nevertheless, so long as $u$ is bounded away from zero, $u$ and $w$ live in the same $H^{n}$ spaces. Specifically:

Lemma 11. Let $T>0$ be given. Let $s \in \mathbf{N}$ such that $s \geq 1$ be given. Assume that $u$ is bounded uniformly away from zero. Then, $u \in C\left([0, T] ; H^{s}\right)$ if and only if $w \in C\left([0, T] ; H^{s}\right)$.

This lemma shows that in order to show existence of solutions for the initial value problem for $K(2,2)$, it is sufficient to show that the initial value problem for $w$ is solvable.

Proof: We will only prove the backward direction of the implication; the proof of the forward implication is the same. Since $u$ is bounded uniformly away from zero, $w$ is also bounded uniformly away from zero. Let $\bar{c}>0$ be given such that for all $x \in X$ and for all $t \in[0, T]$, we have $w(x, t)>\bar{c}$.

Since $w \in C\left([0, T] ; H^{s}\right)$ with $s \geq 1$, by Sobolev imbedding we see $w \in C(X \times$ $[0, T])$, and therefore $u=w^{\beta} \in C(X \times[0, T])$. This clearly implies $u \in C\left([0, T] ; L^{2}\right)$. Since $w(x, t)>\bar{c}>0$ for all $x$ and $t$, we also have that $w^{\beta-1} \in C(X \times[0, T])$. Since $w \in C\left([0, T] ; H^{s}\right)$, we have $w_{x} \in C\left([0, T] ; H^{s-1}\right) \subseteq C\left([0, T] ; L^{2}\right)$. Multiplying, we see that $w^{\beta-1} w_{x} \in C\left([0, T] ; L^{2}\right)$, so $u_{x} \in C\left([0, T] ; L^{2}\right)$, and therefore, $u \in$ $C\left([0, T] ; H^{1}\right)$.

If $s \geq 2$, then similarly, $w^{\beta-1} w_{x x} \in C\left([0, T] ; H^{s-2}\right) \subseteq C\left([0, T] ; L^{2}\right)$. Because $w(x, t)>\bar{c}>0$ for all $x$ and $t$, we have that $w^{\beta-2}$ is continuous on $X \times[0, T]$. By the Sobolev algebra property, $w_{x}^{2} \in C\left([0, T] ; H^{s-1}\right)$, and therefore $w^{\beta-2} w_{x}^{2} \in$ $C\left([0, T] ; H^{s-1}\right) \subseteq C\left([0, T] ; L^{2}\right)$. From the above formula for $u_{x x}$, this implies $u_{x x} \in$ $C\left([0, T] ; L^{2}\right)$, and therefore $u \in C\left([0, T] ; H^{2}\right)$. 
We can continue in this manner for larger $s$; this completes the proof.

Now, we derive the evolution equation for $w$. Using (1) together with the definition of $w$, we have

$$
u_{t}=\beta w^{\beta-1} w_{t}=2 u u_{x x x}+6 u_{x} u_{x x}+2 u u_{x} .
$$

We plug in the above formulas for $u$ and its first three derivatives, and solve for $w_{t}$, finding the following:

(41) $w_{t}=2 w^{\beta} w_{x x x}+(12 \beta-6) w^{\beta-1} w_{x} w_{x x}+(\beta-1)(8 \beta-4) w^{\beta-2} w_{x}^{3}+2 w^{\beta} w_{x}$.

We consider this equation with the initial data

$$
w(x, 0)=w_{0}(x) .
$$

For $n \in \mathbf{N}$ with $n \geq 4$, and for some positive constant $a_{0}$, we assume (similarly as in Assumption 2 the following:

$$
w_{0} \in H^{n} \quad \text { and } \quad w_{0}(x) \geq a_{0} \text {, for all } x \in X .
$$

The $K(2,2)$ equation has the following conserved quantities:

$$
\int_{X} u^{3}(x, t) d x, \quad \int_{X} u(x, t) d x .
$$

The estimates we will be making can be viewed as generalizing the first of these. In particular, we view the first of these conserved quantities as being a conservation law for the $L^{2}$ norm of $u^{3 / 2}$; notice that in the case $n=0$, we have $\beta=2 / 3$, so $w=u^{3 / 2}$. For larger values of $n$, we find that different powers of $u$ can be used to find a short-time estimate in $H^{n}$.

3.1. Uniqueness and continuous dependence for $K(2,2)$. In this section we begin with a continuous dependence result in $L^{2}$. Uniqueness and continuous dependence in more regular spaces will be a corollary of this first result. Since we are making estimates in $L^{2}$, we choose $\beta$ accordingly. Setting $n=0$ in 40 gives

$$
\beta=2 / 3
$$

and we take this value for $\beta$ throughout this subsection.

Theorem 12. Let $w_{1}$ and $w_{2}$ be solutions of 41), with $w_{1}(x, 0)=w_{0}(x)$ and $w_{2}(x, 0)=\widetilde{w}_{0}(x)$, with both of these pieces of initial data satisfying $(43)$. Assume that there exists $T>0$ such that $w_{1} \in L^{\infty}\left([0, T] ; H^{n}\right)$ and $w_{2} \in L^{\infty}\left([0, T] ; H^{n}\right)$. Assume also that for all $t \in[0, T]$, and for all $x \in X$, we have $w_{1}(x, t)>a_{0} / 2$ and $w_{2}(x, t)>a_{0} / 2$. Then there exists $c>0$ such that for all $t \in[0, T]$,

$$
\left\|w_{1}(\cdot, t)-w_{2}(\cdot, t)\right\|_{L^{2}}^{2} \leq\left(\left\|w_{0}-\widetilde{w}_{0}\right\|_{L^{2}}^{2}\right) e^{c t} .
$$

Proof: Define

$$
E_{d}(t)=\frac{1}{2} \int_{X}\left(w_{1}(x, t)-w_{2}(x, t)\right)^{2} d x .
$$

Differentiating with respect to time, we find

$$
\frac{d E_{d}}{d t}=\int_{X}\left(w_{1}-w_{2}\right)\left(w_{1, t}-w_{2, t}\right) d x .
$$

We plug in from the evolution equation:

$$
\frac{d E_{d}}{d t}=A_{1}+A_{2}+A_{3}+A_{4}
$$


where

and

$$
\begin{gathered}
A_{1}=\int_{X} 2\left(w_{1}-w_{2}\right)\left(w_{1}^{\beta} w_{1, x x x}-w_{2}^{\beta} w_{2, x x x}\right) d x \\
A_{2}=\int_{X}(12 \beta-6)\left(w_{1}-w_{2}\right)\left(w_{1}^{\beta-1} w_{1, x} w_{1, x x}-w_{2}^{\beta-1} w_{2, x} w_{2, x x}\right) d x \\
A_{3}=\int_{X}(\beta-1)(8 \beta-4)\left(w_{1}-w_{2}\right)\left(w_{1}^{\beta-2} w_{1, x}^{3}-w_{2}^{\beta-2} w_{2, x}^{3}\right) d x,
\end{gathered}
$$

$$
A_{4}=\int_{X} 2\left(w_{1}-w_{2}\right)\left(w_{1}^{\beta} w_{1, x}-w_{2}^{\beta} w_{2, x}\right) d x .
$$

We begin by adding and subtracting in $A_{1}$ :

$$
\begin{aligned}
A_{1}=\int_{X} 2\left(w_{1}-w_{2}\right)\left(w_{1}^{\beta}\right. & \left.-w_{2}^{\beta}\right) w_{1, x x x} d x \\
& +\int_{X} 2\left(w_{1}-w_{2}\right)\left(w_{2}^{\beta}\right)\left(w_{1, x x x}-w_{2, x x x}\right) d x=: B_{1}+B_{2} .
\end{aligned}
$$

On the domain of interest, the function $g(z)=z^{\beta}$ is Lipschitz continuous, so we have

$$
B_{1} \leq c E_{d}
$$

For $B_{2}$, we integrate by parts:

$$
\begin{aligned}
B_{2}=-\int_{X} 2\left(w_{1, x}-w_{2, x}\right)\left(w_{2}^{\beta}\right) & \left(w_{1, x x}-w_{2, x x}\right) d x \\
& -\int_{X} 2 \beta\left(w_{1}-w_{2}\right)\left(w_{2}^{\beta-1} w_{2, x}\right)\left(w_{1, x x}-w_{2, x x}\right) d x .
\end{aligned}
$$

We recognize that the first term on the right-hand side includes a perfect derivative, and we integrate by parts again:

$$
\begin{aligned}
B_{2}=\int_{X} \beta\left(w_{2}^{\beta-1} w_{2, x}\right)\left(w_{1, x}-\right. & \left.w_{2, x}\right)^{2} d x \\
& -\int_{X} 2 \beta\left(w_{1}-w_{2}\right)\left(w_{2}^{\beta-1} w_{2, x}\right)\left(w_{1, x x}-w_{2, x x}\right) d x .
\end{aligned}
$$

We integrate the second term on the right-hand side by parts:

$$
\begin{aligned}
B_{2}=\int_{X} 3 \beta\left(w_{2}^{\beta-1} w_{2, x}\right)\left(w_{1, x}\right. & \left.-w_{2, x}\right)^{2} d x \\
& +\int_{X} 2 \beta\left(w_{1}-w_{2}\right)\left(w_{2}^{\beta-1} w_{2, x}\right)_{x}\left(w_{1, x}-w_{2, x}\right) d x .
\end{aligned}
$$

For the second term on the right-hand side, we again notice a perfect derivative, and integrate by parts:

$$
B_{2}=\int_{X} 3 \beta\left(w_{2}^{\beta-1} w_{2, x}\right)\left(w_{1, x}-w_{2, x}\right)^{2} d x-\int_{X} \beta\left(w_{2}^{\beta-1} w_{2, x}\right)_{x x}\left(w_{1}-w_{2}\right)^{2} d x .
$$

We now consider $A_{2}$. To begin, we add and subtract:

$$
\begin{aligned}
A_{2}=\int_{X}( & 12 \beta-6)\left(w_{1}-w_{2}\right)\left(w_{1}^{\beta-1} w_{1, x}-w_{2}^{\beta-1} w_{2, x}\right) w_{1, x x} d x \\
& \quad+\int_{X}(12 \beta-6)\left(w_{1}-w_{2}\right)\left(w_{2}^{\beta-1} w_{2, x}\right)\left(w_{1, x x}-w_{2, x x}\right) d x=: B_{3}+B_{4} .
\end{aligned}
$$


To estimate $B_{3}$, we add and subtract once more:

$$
\begin{aligned}
B_{3}=\int_{X}(12 \beta-6)\left(w_{1}-\right. & \left.w_{2}\right)\left(w_{1}^{\beta-1}-w_{2}^{\beta-1}\right) w_{1, x} w_{1, x x} d x \\
& +\int_{X}(12 \beta-6)\left(w_{1}-w_{2}\right)\left(w_{2}^{\beta-1}\right)\left(w_{1, x}-w_{2, x}\right) w_{1, x x} d x .
\end{aligned}
$$

For the second term on the right-hand side, we recognize the presence of a perfect derivative, and we integrate by parts:

$$
\begin{aligned}
B_{3}=\int_{X}(12 \beta-6)\left(w_{1}-w_{2}\right)\left(w_{1}^{\beta-1}-\right. & \left.w_{2}^{\beta-1}\right) w_{1, x} w_{1, x x} d x \\
& -\int_{X}(6 \beta-3)\left(w_{2}^{\beta-1} w_{1, x x}\right)_{x}\left(w_{1}-w_{2}\right)^{2} d x .
\end{aligned}
$$

Using the uniform bounds, and the fact that the function $g(z)=z^{\beta-1}$ is Lipschitz on the relevant domain, we find

$$
B_{3} \leq c E_{d}
$$

For $B_{4}$, we integrate by parts:

$$
\begin{aligned}
B_{4}=-\int_{X}(12 \beta-6)\left(w_{2}^{\beta-1} w_{2, x}\right)\left(w_{1, x}-w_{2, x}\right)^{2} d x & \\
& -\int_{X}(12 \beta-6)\left(w_{1}-w_{2}\right)\left(w_{2}^{\beta-1} w_{2, x}\right)_{x}\left(w_{1, x}-w_{2, x}\right) d x .
\end{aligned}
$$

For the second term on the right-hand side, we recognize a perfect derivative and we integrate by parts:

$$
\begin{aligned}
B_{4}=-\int_{X}(12 \beta-6)\left(w_{2}^{\beta-1} w_{2, x}\right)\left(w_{1, x}\right. & \left.-w_{2, x}\right)^{2} d x \\
& +\int_{X}(6 \beta-3)\left(w_{2}^{\beta-1} w_{2, x}\right)_{x x}\left(w_{1}-w_{2}\right)^{2} d x .
\end{aligned}
$$

Now, since we have $\beta=2 / 3$, and if we add $A_{1}+A_{2}$, there is an important cancellation, and we find

$$
A_{1}+A_{2}=B_{1}+B_{2}+B_{3}+B_{4} \leq c E_{d} .
$$

We can continue in the same manner (adding and subtracting, recognizing perfect derivatives, and integrating by parts), and we will find

$$
A_{3} \leq c E_{d}, \quad A_{4} \leq c E_{d} .
$$

Altogether, we have found

$$
\frac{d E_{d}}{d t} \leq c E_{d}
$$

This clearly implies

$$
E_{d}(t) \leq E_{d}(0) e^{c t} .
$$

This completes the proof of the lemma.

We can use this result to prove uniqueness, and we can also use it to get continuous dependence on the initial data in more regular spaces. 
Corollary 13. Let $w_{1}$ and $w_{2}$ be solutions of 411), with $w_{1}(x, 0)=w_{0}(x)$ and $w_{2}(x, 0)=\widetilde{w}_{0}(x)$, with the initial data satisfying (43). Assume that there exists $T>0$ such that $w_{1} \in L^{\infty}\left([0, T] ; H^{n}\right)$ and $w_{2} \in L^{\infty}\left([0, T] ; H^{n}\right)$. Assume also that for all $t \in[0, T]$, and for all $x \in X$, we have $w_{1}(x, t)>a_{0} / 2$ and $w_{2}(x, t)>a_{0} / 2$. Let $n^{\prime}$ be given such that $0 \leq n^{\prime}<n$. Then, there exists a constant $C>0$ such that for all $t \in[0, T]$,

$$
\left\|w_{1}(\cdot, t)-w_{2}(\cdot, t)\right\|_{H^{n^{\prime}}} \leq C\left\|w_{0}-\widetilde{w}_{0}\right\|_{L^{2}}^{1-n^{\prime} / n} .
$$

Proof: This is a straightforward combination of Theorem 12 and the Sobolev interpolation inequality used in (26). This completes the proof.

Corollary 14. Let $w_{1}$ and $w_{2}$ be solutions of (41), with $w_{1}(x, 0)=w_{0}(x)$ and $w_{2}(x, 0)=\widetilde{w}_{0}(x)$, with the initial data satisfying (43). Assume that there exists $T>0$ such that $w_{1} \in L^{\infty}\left([0, T] ; H^{n}\right)$ and $w_{2} \in L^{\infty}\left([0, T] ; H^{n}\right)$. Assume also that for all $t \in[0, T]$, and for all $x \in X$, we have $w_{1}(x, t)>a_{0} / 2$ and $w_{2}(x, t)>a_{0} / 2$. If $w_{0}=\widetilde{w}_{0}$, then for all $t \in[0, T], w_{1}(\cdot, t)=w_{2}(\cdot, t)$.

Proof: This follows immediately from Theorem 12 .

Remark 7. In light of Lemma 11, the uniqueness and continuous dependence theorems we have proved for $w$ also give uniqueness and continuous dependence results for solutions of the original $K(2,2)$ equation, (1).

3.2. Existence of solutions for $K(2,2)$. We now need to work in the regularity space of the initial data, $H^{n}$. Recall $n \in \mathbf{N}$, and $n \geq 4$. Take $\beta$ as in 40, that is $\beta=\frac{6}{2 n+9}$.

We introduce a mollifed version of (41). Specifically take $\mathcal{J}_{\epsilon}$, for $\epsilon>0$, to be the same mollifier as was described in Theorem 8. Our mollified equation is

$$
\begin{array}{r}
w_{t}^{\epsilon}=\mathcal{J}_{\epsilon}\left[2\left(\mathcal{J}_{\epsilon} w^{\epsilon}\right)^{\beta}\left(\mathcal{J}_{\epsilon} w_{x x x}^{\epsilon}\right)+(12 \beta-6)\left(\mathcal{J}_{\epsilon} w^{\epsilon}\right)^{\beta-1}\left(\mathcal{J}_{\epsilon} w_{x}^{\epsilon}\right)\left(\mathcal{J}_{\epsilon} w_{x x}^{\epsilon}\right)\right. \\
\left.+(\beta-1)(8 \beta-4)\left(\mathcal{J}_{\epsilon} w^{\epsilon}\right)^{\beta-2}\left(\mathcal{J}_{\epsilon} w_{x}^{\epsilon}\right)^{3}+2\left(\mathcal{J}_{\epsilon} w^{\epsilon}\right)^{\beta}\left(\mathcal{J}_{\epsilon} w_{x}^{\epsilon}\right)\right] .
\end{array}
$$

This evolution equation is taken with the initial condition

$$
w^{\epsilon}(x, 0)=w_{0}(x),
$$

where $w_{0} \in H^{n}$ and there exists $a_{0}>0$ such that for all $x \in X, w_{0}(x)>a_{0}$. We will seek solutions of this initial value problem which satisfy at positive times $t$ the condition

$$
w^{\epsilon}(x, t)>\frac{a_{0}}{2} \text { for all } x \in X .
$$

Given $w_{0} \in H^{n}$ which satisfies the condition $w_{0}(x)>a_{0}$ for all $x \in X$, we define an open set $\mathcal{O} \subset H^{n}$ such that $w_{0} \in \mathcal{O}$. Specifically

$$
\mathcal{O}:=\left\{f \in H^{n}:\|f\|_{H^{n}}<2\left\|w_{0}\right\|_{H^{n}} \text { and, for all } x \in X,|f(x)|>\frac{a_{0}}{2}\right\} .
$$

The following existence result is a direct application of the Picard theorem, and we note it without further proof. 
Lemma 15. For any $\epsilon>0$, there exists $T_{\epsilon}>0$ such that there exists $w^{\epsilon}(x, t) \epsilon$ $C^{1}\left(\left[-T_{\epsilon}, T_{\epsilon}\right] ; \mathcal{O}\right)$ such that $w^{\epsilon}$ is a solution of the initial value problem 444,4 , 45 .

Remark 8. To be clear, for all $t \in\left[-T_{\epsilon}, T_{\epsilon}\right]$, since $w^{\epsilon}(\cdot, t) \in \mathcal{O}$, the solution $w^{\epsilon}$ satisfies the condition 46.

The time of existence in Lemma 15 depends badly on $\epsilon$. In order to remove this dependence, we will perform an $H^{n}$ energy estimate for this equation; this will allow us to use the continuation theorem for autonomous differential equations. Our energy is the following:

$$
E(t)=\frac{1}{2} \int_{X}\left(w^{\epsilon}\right)^{2} d x+\frac{1}{2} \int_{X}\left(\partial_{x}^{n} w^{\epsilon}\right)^{2} d x:=E_{0}+E_{n} .
$$

Since we are estimating $w^{\epsilon}$ in $H^{n}$, it will be helpful to apply $n$ spatial derivatives to 44):

$$
\begin{array}{r}
\partial_{x}^{n} w_{t}^{\epsilon}=\mathcal{J}_{\epsilon}\left[2\left(\mathcal{J}_{\epsilon} w^{\epsilon}\right)^{\beta} \partial_{x}^{n+3} \mathcal{J}_{\epsilon} w^{\epsilon}+(12 \beta+2 \beta n-6)\left(\mathcal{J}_{\epsilon} w^{\epsilon}\right)^{\beta-1}\left(\mathcal{J}_{\epsilon} w_{x}^{\epsilon}\right) \partial_{x}^{n+2} \mathcal{J}_{\epsilon} w^{\epsilon}\right. \\
\left.+F_{1}\left[\mathcal{J}_{\epsilon} w^{\epsilon}\right] \partial_{x}^{n+1} \mathcal{J}_{\epsilon} w^{\epsilon}+F_{2}\left[\mathcal{J}_{\epsilon} w^{\epsilon}\right]\right]
\end{array}
$$

Formulas and estimates for $F_{1}$ and $F_{2}$ are the subject of the next subsection.

3.2.1. The Lower-Order Terms. The formula for $F_{1}$ is

$$
\begin{aligned}
& (48) \quad F_{1}\left[\mathcal{J}_{\epsilon} w^{\epsilon}\right]=2\left(\begin{array}{c}
n \\
2
\end{array}\right)\left(\left(\mathcal{J}_{\epsilon} w^{\epsilon}\right)^{\beta}\right)_{x x}+(12 \beta-6)\left(\mathcal{J}_{\epsilon} w^{\epsilon}\right)^{\beta-1} \mathcal{J}_{\epsilon} w_{x x}^{\epsilon} \\
& +(12 \beta-6) n \partial_{x}\left(\left(\mathcal{J}_{\epsilon} w^{\epsilon}\right)^{\beta-1} \mathcal{J}_{\epsilon} w_{x}^{\epsilon}\right)+3(\beta-1)(8 \beta-4)\left(\mathcal{J}_{\epsilon} w^{\epsilon}\right)^{\beta-2}\left(\mathcal{J}_{\epsilon} w_{x}^{\epsilon}\right)^{2}+2\left(\mathcal{J}_{\epsilon} w^{\epsilon}\right)^{\beta} .
\end{aligned}
$$

Upon expanding the various derivatives in the above formula, we find

$$
F_{1}=d_{1}\left(\mathcal{J}_{\epsilon} w^{\epsilon}\right)^{\beta-2}\left(\mathcal{J}_{\epsilon} w_{x}^{\epsilon}\right)^{2}+d_{2}\left(\mathcal{J}_{\epsilon} w^{\epsilon}\right)^{\beta-1}\left(\mathcal{J}_{\epsilon} w^{\epsilon}\right)_{x x}+2\left(\mathcal{J}_{\epsilon} w^{\epsilon}\right)^{\beta},
$$

for some constants $d_{1}$ and $d_{2}$.

Recall that we are considering $w^{\epsilon}$ such that for all $x$ and $t, w^{\epsilon}(x, t)>0$. Since $\mathcal{J}_{\epsilon}$ is an averaging operator, for any $z \in X$ and for any $t$ we have $\inf _{x \in X} w^{\epsilon}(x, t) \leq$ $\mathcal{J}_{\epsilon} w^{\epsilon}(z, t)$, and therefore

$$
\left\|\frac{1}{\mathcal{J}_{\epsilon} w^{\epsilon}(z)}\right\|_{L^{\infty}} \leq\left\|\frac{1}{w^{\epsilon}}\right\|_{L^{\infty}} .
$$

Since $\beta<1$, we have $\beta-1<0$, and $\beta-2<0$, and so on. We then estimate, for instance,

$$
\left|\mathcal{J}_{\epsilon} w^{\epsilon}\right|^{\beta-1} \leq\left\|\frac{1}{w^{\epsilon}}\right\|_{L^{\infty}}^{1-\beta} .
$$

We estimate other negative powers of $\mathcal{J}_{\epsilon} w^{\epsilon}$ in the same way.

We therefore have the following bounds for $F_{1}$ :

$$
\left|F_{1}\right|_{L^{\infty}} \leq \gamma_{1}\left(\left\|\frac{1}{w^{\epsilon}}\right\|_{L^{\infty}}\right)\left(\left\|w^{\epsilon}\right\|_{3}^{2}+\left\|w^{\epsilon}\right\|_{3}^{\beta}\right) \leq \gamma_{1}\left(\left\|\frac{1}{w^{\epsilon}}\right\|_{L^{\infty}}\right)\left(E+E^{\beta / 2}\right),
$$




$$
\left|\partial_{x} F_{1}\right|_{L^{\infty}} \leq \gamma_{2}\left(\left\|\frac{1}{w^{\epsilon}}\right\|_{L^{\infty}}\right)\left(\left\|w^{\epsilon}\right\|_{4}^{3}+\left\|w^{\epsilon}\right\|_{4}\right) \leq \gamma_{2}\left(\left\|\frac{1}{w^{\epsilon}}\right\|_{L^{\infty}}\right)\left(E^{3 / 2}+E^{1 / 2}\right),
$$

for some smooth functions $\gamma_{1}$ and $\gamma_{2}$. Note: we only actually will need the second of these two bounds.

For $F_{2}$, we clearly have the definition

$$
\begin{gathered}
F_{2}\left[\mathcal{J}_{\epsilon} w^{\epsilon}\right]=\partial_{x}^{n}\left[2\left(\mathcal{J}_{\epsilon} w^{\epsilon}\right)^{\beta}\left(\mathcal{J}_{\epsilon} w_{x x x}^{\epsilon}\right)+(12 \beta-6)\left(\mathcal{J}_{\epsilon} w^{\epsilon}\right)^{\beta-1}\left(\mathcal{J}_{\epsilon} w_{x}^{\epsilon}\right)\left(\mathcal{J}_{\epsilon} w_{x x}^{\epsilon}\right)\right. \\
\left.+(\beta-1)(8 \beta-4)\left(\mathcal{J}_{\epsilon} w^{\epsilon}\right)^{\beta-2}\left(\mathcal{J}_{\epsilon} w_{x}^{\epsilon}\right)^{3}+2\left(\mathcal{J}_{\epsilon} w^{\epsilon}\right)^{\beta}\left(\mathcal{J}_{\epsilon} w_{x}^{\epsilon}\right)\right] \\
-\left[2\left(\mathcal{J}_{\epsilon} w^{\epsilon}\right)^{\beta} \partial_{x}^{n+3} \mathcal{J}_{\epsilon} w^{\epsilon}+(12 \beta+2 \beta n-6)\left(\mathcal{J}_{\epsilon} w^{\epsilon}\right)^{\beta-1}\left(\mathcal{J}_{\epsilon} w_{x}^{\epsilon}\right) \partial_{x}^{n+2} \mathcal{J}_{\epsilon} w^{\epsilon}\right. \\
\left.+F_{1}\left[\mathcal{J}_{\epsilon} w^{\epsilon}\right] \partial_{x}^{n+1} \mathcal{J}_{\epsilon} w^{\epsilon}\right]
\end{gathered}
$$

For ease of estimating, it is helpful to rewrite this using the product rule:

$$
\begin{gathered}
F_{2}\left[\mathcal{J}_{\epsilon} w^{\epsilon}\right]=2 \sum_{k=3}^{n}\left(\begin{array}{l}
n \\
k
\end{array}\right)\left(\partial_{x}^{k}\left(\left(\mathcal{J}_{\epsilon} w^{\epsilon}\right)^{\beta}\right)\right)\left(\partial_{x}^{n-k+3} \mathcal{J}_{\epsilon} w^{\epsilon}\right) \\
+(12 \beta-6) \sum_{k=2}^{n-1}\left(\begin{array}{l}
n \\
k
\end{array}\right)\left(\partial_{x}^{k}\left(\left(\mathcal{J}_{\epsilon} w^{\epsilon}\right)^{\beta-1} \mathcal{J}_{\epsilon} w_{x}^{\epsilon}\right)\right)\left(\partial_{x}^{n-k+2} \mathcal{J}_{\epsilon} w^{\epsilon}\right) \\
+(12 \beta-6) \sum_{k=1}^{n}\left(\begin{array}{c}
n \\
k
\end{array}\right)\left(\mathcal{J}_{\epsilon} w_{x x}^{\epsilon}\right)\left(\partial_{x}^{k}\left(\mathcal{J}_{\epsilon} w^{\epsilon}\right)^{\beta-1}\right)\left(\partial_{x}^{n-k+1} \mathcal{J}_{\epsilon} w^{\epsilon}\right) \\
+3(\beta-1)(8 \beta-4) \sum_{k=1}^{n-1}\left(\begin{array}{c}
n-1 \\
k
\end{array}\right)\left(\partial_{x}^{k}\left(\left(\mathcal{J}_{\epsilon} w^{\epsilon}\right)^{\beta-2}\left(\mathcal{J}_{\epsilon} w_{x}^{\epsilon}\right)^{2}\right)\right)\left(\partial_{k}^{n+1-k} w^{\varepsilon}\right) \\
+(\beta-1)(8 \beta-4) \sum_{k=0}^{n-1}\left(\begin{array}{c}
n-1 \\
k
\end{array}\right)\left(\partial_{x}^{k+1}\left(\mathcal{J}_{\epsilon} w^{\epsilon}\right)^{\beta-2}\right)\left(\partial_{x}^{n-1-k}\left(\mathcal{J}_{\epsilon} w_{x}^{\epsilon}\right)^{3}\right) \\
+2 \sum_{k=1}^{n}\left(\begin{array}{l}
n \\
k
\end{array}\right)\left(\partial_{x}^{k}\left(\left(\mathcal{J}_{\epsilon} w^{\epsilon}\right)^{\beta}\right)\right)\left(\partial_{x}^{n-k+1} \mathcal{J}_{\epsilon} w^{\epsilon}\right) .
\end{gathered}
$$

We now give an estimate for $F_{2}$ in $L^{2}$. For this estimate, note that the highest number of derivatives on $w^{\epsilon}$ that appear anywhere in (51) is $n$. So, $F_{2}$ will be bounded in terms of $E$, but we should be careful as to which powers of $E$ can be used for the bound, and we need to be careful here because we estimate positive powers and negative powers differently. Notice that in the fifth summation on the right-hand side of (51), the $k=n-1$ term includes a factor $\partial_{x}^{n}\left(\left(\mathcal{J}_{\epsilon} w^{\epsilon}\right)^{\beta-2}\right)$. In this factor, if we apply the derivatives by using the chain rule and the product rule, then we find one term which has as a factor $\left(\mathcal{J}_{\epsilon} w^{\epsilon}\right)^{\beta-2-n}$. This is the most negative power encountered of all the terms in 51). Similarly, we want to identify the least negative power on the right-hand side of (51); this comes, for instance, from the $k=1$ term in the sixth summation, and is $\left(\mathcal{J}_{\epsilon} w^{\epsilon}\right)^{\beta-1}$. From these considerations, 
we find the following estimate:

$$
\left\|F_{2}\right\|_{L^{2}} \leq \gamma_{3}\left(\left\|\frac{1}{w^{\epsilon}}\right\|_{L^{\infty}}\right)\left(\left\|w^{\epsilon}\right\|_{H^{n}}^{n+3}+\left\|w^{\epsilon}\right\|_{H^{n}}^{2}\right) \leq \gamma_{3}\left(\left\|\frac{1}{w^{\epsilon}}\right\|_{L^{\infty}}\right)\left(E^{(n+3) / 2}+E\right),
$$

for some smooth function $\gamma_{3}$.

3.2.2. The Energy Estimate. The goal of this section is to prove the following lemma:

Lemma 16. Let $\epsilon>0$ be given. Assume there exists $\bar{T}>0$ such that there exists $w^{\epsilon} \in C\left([0, \bar{T}] ; H^{n}\right)$ which satisfies (44) and (46). Then there exist positive constants $C_{1}$ and $C_{2}$ which depend only on $a_{0}$ and $n$ such that for all $t \in[0, \bar{T}]$, we have

$$
E(t) \leq-\frac{\ln \left(\exp \left\{-C_{2} E(0)\right\}-C_{1} C_{2} t\right)}{C_{2}} .
$$

Proof: The method of proof is to first prove an estimate for the growth of the energy. This provides a differential inequality, and the solution of this inequality gives the estimate we are trying to establish.

Clearly, the growth of $E_{0}$ is bounded by $E$ :

$$
\frac{d E_{0}}{d t} \leq \gamma_{4}\left(\left\|\frac{1}{w^{\epsilon}}\right\|_{L^{\infty}}\right)\left(E^{1+\beta / 2}+E^{2}\right)
$$

for some smooth $\gamma_{4}$.

We consider now the growth of $E_{n}$ :

$$
\begin{aligned}
\frac{d E_{n}}{d t}=F+ & \int_{X} 2\left(\mathcal{J}_{\epsilon} w^{\epsilon}\right)^{\beta}\left(\partial_{x}^{n} \mathcal{J}_{\epsilon} w^{\epsilon}\right)\left(\partial_{x}^{n+3} \mathcal{J}_{\epsilon} w^{\epsilon}\right) \\
& +(12 \beta+2 \beta n-6)\left(\mathcal{J}_{\epsilon} w^{\epsilon}\right)^{\beta-1}\left(\mathcal{J}_{\epsilon} w_{x}^{\epsilon}\right)\left(\partial_{x}^{n} \mathcal{J}_{\epsilon} w^{\epsilon}\right)\left(\partial_{x}^{n+2} \mathcal{J}_{\epsilon} w^{\epsilon}\right) d x
\end{aligned}
$$

where we have introduced the term

$$
F=\int_{X}\left(F_{1}\left[\mathcal{J}_{\epsilon} w^{\epsilon}\right] \partial_{x}^{n+1} \mathcal{J}_{\epsilon} w^{\epsilon}+F_{2}\left[\mathcal{J}_{\epsilon} w^{\epsilon}\right]\right)\left(\partial_{x}^{n} \mathcal{J}_{\epsilon} w^{\epsilon}\right) d x
$$

We can bound $F$ in terms of the energy easily; first, this requires one integration by parts:

$$
F=\int_{X}-\frac{1}{2}\left(F_{1}\left[\mathcal{J}_{\epsilon} w^{\epsilon}\right]\right)_{x}\left(\partial_{x}^{n} \mathcal{J}_{\epsilon} w^{\epsilon}\right)^{2}+\left(F_{2}\left[\mathcal{J}_{\epsilon} w^{\epsilon}\right]\right)\left(\partial_{x}^{n} \mathcal{J}_{\epsilon} w^{\epsilon}\right) d x
$$

By the above bounds 49 and 52 for the $F_{i}$, and since $n \geq 4$, we have

$$
|F| \leq \gamma_{5}\left(\left\|\frac{1}{w^{\epsilon}}\right\|_{L^{\infty}}\right)\left(E^{(n+4) / 2}+E^{3 / 2}\right)
$$

for some smooth $\gamma_{5}$.

We integrate by parts in the first term on the right-hand side of 53):

$$
\begin{aligned}
\frac{d E_{n}}{d t}=F+ & \int_{X}\left[-2\left(\mathcal{J}_{\epsilon} w^{\epsilon}\right)^{\beta}\left(\partial_{x}^{n+1} \mathcal{J}_{\epsilon} w^{\epsilon}\right)\left(\partial_{x}^{n+2} \mathcal{J}_{\epsilon} w^{\epsilon}\right)\right. \\
& \left.+(10 \beta+2 \beta n-6)\left(\mathcal{J}_{\epsilon} w^{\epsilon}\right)^{\beta-1}\left(\mathcal{J}_{\epsilon} w_{x}^{\epsilon}\right)\left(\partial_{x}^{n} \mathcal{J}_{\epsilon} w^{\epsilon}\right)\left(\partial_{x}^{n+2} \mathcal{J}_{\epsilon} w^{\epsilon}\right)\right] d x
\end{aligned}
$$


We recognize that the first term in the integrand on the right-hand side of (54) includes a perfect derivative, and we integrate it by parts:

$$
\begin{aligned}
\frac{d E_{n}}{d t}=F+ & \int_{X}\left[\beta\left(\mathcal{J}_{\epsilon} w^{\epsilon}\right)^{\beta-1}\left(\mathcal{J}_{\epsilon} w_{x}^{\epsilon}\right)\left(\partial_{x}^{n+1} \mathcal{J}_{\epsilon} w^{\epsilon}\right)^{2}\right. \\
& \left.+(10 \beta+2 \beta n-6)\left(\mathcal{J}_{\epsilon} w^{\epsilon}\right)^{\beta-1}\left(\mathcal{J}_{\epsilon} w_{x}^{\epsilon}\right)\left(\partial_{x}^{n} \mathcal{J}_{\epsilon} w^{\epsilon}\right)\left(\partial_{x}^{n+2} \mathcal{J}_{\epsilon} w^{\epsilon}\right)\right] d x
\end{aligned}
$$

Now, we integrate the second term in the integrand on the right-hand side of 55 ) by parts:

$$
\begin{aligned}
\frac{d E_{n}}{d t}= & F+\int_{X}\left[(-9 \beta-2 \beta n+6)\left(\mathcal{J}_{\epsilon} w^{\epsilon}\right)^{\beta-1}\left(\mathcal{J}_{\epsilon} w_{x}^{\epsilon}\right)\left(\partial_{x}^{n+1} \mathcal{J}_{\epsilon} w^{\epsilon}\right)^{2}\right. \\
& \left.-(10 \beta+2 \beta n-6)\left(\left(\mathcal{J}_{\epsilon} w^{\epsilon}\right)^{\beta-1}\left(\mathcal{J}_{\epsilon} w_{x}^{\epsilon}\right)\right)_{x}\left(\partial_{x}^{n} \mathcal{J}_{\epsilon} w^{\epsilon}\right)\left(\partial_{x}^{n+1} \mathcal{J}_{\epsilon} w^{\epsilon}\right)\right] d x
\end{aligned}
$$

Since $\beta=\frac{6}{2 n+9}$, the first term in the integrand on the right-hand side of 56 is zero (this is the reason for selecting $\beta$ this way):

$$
\frac{d E_{n}}{d t}=F-\int_{X}(10 \beta+2 \beta n-6)\left(\left(\mathcal{J}_{\epsilon} w^{\epsilon}\right)^{\beta-1}\left(\mathcal{J}_{\epsilon} w_{x}^{\epsilon}\right)\right)_{x}\left(\partial_{x}^{n} \mathcal{J}_{\epsilon} w^{\epsilon}\right)\left(\partial_{x}^{n+1} \mathcal{J}_{\epsilon} w^{\epsilon}\right) d x
$$

We integrate by parts in the right-hand side of (57) once more:

$$
\frac{d E_{n}}{d t}=F+\int_{X}(5 \beta+\beta n-3)\left(\left(\mathcal{J}_{\epsilon} w^{\epsilon}\right)^{\beta-1}\left(\mathcal{J}_{\epsilon} w_{x}^{\epsilon}\right)\right)_{x x}\left(\partial_{x}^{n} \mathcal{J}_{\epsilon} w^{\epsilon}\right)^{2} d x
$$

Finally, we have

$$
\frac{d E}{d t} \leq \gamma_{6}\left(\left\|\frac{1}{w^{\epsilon}}\right\|_{L^{\infty}}\right)\left(E^{(n+4) / 2}+E^{3 / 2}\right)
$$

for some smooth $\gamma_{6}$.

Since condition 46 is satisfied, we can rewrite 59 as

$$
\frac{d E}{d t} \leq \bar{c}\left(E^{(n+4) / 2}+E^{3 / 2}\right) .
$$

There exist positive constants $C_{1}$ and $C_{2}$ such that

$$
\bar{c}\left(E^{(n+4) / 2}+E^{3 / 2}\right) \leq C_{1} \exp \left\{C_{2} E\right\},
$$

and these constants depend only on $a_{0}$ and $s$. So, we have

$$
\frac{d E}{d t} \leq C_{1} \exp \left\{C_{2} E\right\} .
$$

This differential inequality can be solved, with the result that

$$
E(t) \leq-\frac{\ln \left(\exp \left(-C_{2} E(0)\right)-C_{1} C_{2} t\right)}{C_{2}} .
$$

This completes the proof. 
3.2.3. Passage to the limit. We now introduce another open set, $\widetilde{\mathcal{O}} \subset H^{n}$ which is defined as:

$$
\widetilde{\mathcal{O}}:=\left\{f \in H^{n}:\|f\|_{H^{n}}<\frac{3}{2}\left\|w_{0}\right\|_{H^{n}} \text { and, for all } x \in X,|f(x)|>\frac{3 a_{0}}{4} .\right\}
$$

Clearly, $\widetilde{\mathcal{O}} \subseteq \mathcal{O}$.

Lemma 17. Let $w_{0} \in H^{n}$ satisfy (46). There exists $T^{*}>0$ such that for all $\epsilon \in(0,1)$, the solution $w^{\epsilon}$ of the initial value problem (44), (45) satisfies $w^{\epsilon} \in$ $C\left(\left[0, T^{*}\right] ; \widetilde{\mathcal{O}}\right)$.

Proof: Assume there does not exist such a $T^{*}$. This implies that there exists a sequence $\epsilon_{j} \rightarrow 0$ such that the solutions $w_{\epsilon_{j}}$ leave the set $\widetilde{\mathcal{O}}$ arbitrarily quickly as $j \rightarrow \infty$. For each $j$, let $T_{j}$ be the infimum of the set of times at which $w_{\epsilon_{j}}$ is not in $\widetilde{\mathcal{O}}$. From Lemma 15 , we know $T_{j}>0$ for all $j$, and by choice of the sequence, we have $\lim _{j \rightarrow \infty} T_{j}=0$. So, for each $j$, we have $w_{\epsilon_{j}} \in C\left(\left[0, T_{j}\right) ; \widetilde{\mathcal{O}}\right)$, and $w_{\epsilon_{j}}\left(\cdot, T_{j}\right) \notin \widetilde{\mathcal{O}}$. Note, however, that we do have $w_{\epsilon_{j}} \in C\left(\left[0, T_{j}\right] ; \mathcal{O}\right)$. In particular, we must have either

$$
\inf _{x \in X} w_{\epsilon_{j}}\left(x, T_{j}\right)=\frac{3 a_{0}}{4}, \quad \text { or } \quad E\left(T_{j}\right)=\frac{9}{4} E(0) .
$$

But this is impossible, from the above uniform estimate. In particular, if we let

$$
\tilde{T}=\frac{\exp \left(-C_{2} E(0)\right)-\exp \left(-2 C_{2} E(0)\right)}{C_{1} C_{2}},
$$

then we see that if $T_{j} \in[0, \tilde{T}]$, then we have

$$
E\left(T_{j}\right) \leq 2 E(0) \text {. }
$$

So, we cannot have $E\left(T_{j}\right)=\frac{9}{4} E(0)$ with $T_{j} \rightarrow 0$.

From the evolution equation for $w^{\epsilon}$, we see that there exists $C>0$ such that for all $\epsilon \in(0,1)$, we have $\left|w_{t}^{\epsilon}\right| \leq C$ as long as $w^{\epsilon} \in \mathcal{O}$. Therefore, if the infimum of $w^{\epsilon}$ was originally at least $a_{0}$, it could not become equal to $\frac{3 a_{0}}{4}$ arbitrarily quickly. This completes the proof.

Theorem 18. Let $w_{0} \in H^{n}$ satisfy (46). Then, there exists $T>0$ such that the initial value problem (44), (45) has a solution $w \in C([0, T] ; \mathcal{O})$.

Proof: We let $T$ equal the value $T^{*}$ from Lemma 17 . For $\epsilon \in(0,1)$, the functions $w^{\epsilon}$ are all in the space $C([0, T] ; \mathcal{O})$, which implies that for all $\epsilon \in(0,1)$, for all $t \in[0, T]$, we have the uniform bound $\left\|w^{\epsilon}(\cdot, t)\right\|_{H^{n}} \leq 2\left\|w_{0}\right\|_{H^{n}}$. Since $s \geq 4$, this implies that there exists $C>0$ such that for all $\epsilon \in(0,1)$, for all $t \in[0, T]$, for all $x \in X$, we have

$$
\left|w^{\epsilon}(x, t)\right| \leq C, \quad\left|w_{x}^{\epsilon}(x, t)\right| \leq C, \quad\left|w_{t}^{\epsilon}(x, t)\right| \leq C .
$$

This means that the family $w^{\epsilon}$ is uniformly bounded and equicontinuous, on the domain $X \times[0, T]$. The Arzela-Ascoli theorem gives a uniform limit of the sequence $w^{\epsilon}$ (taking a subsequence, which we do not relabel) in $C(X \times[0, T])$. Together with the uniform bound and an elementary interpolation inequality, this implies that the limit, $w$, is in $C\left([0, T] ; H^{n^{\prime}}\right)$, for any $0 \leq n^{\prime}<n$.

Furthermore, pointwise in time, $w^{\epsilon}$ is uniformly bounded in $H^{n}$, so there is a weak limit of a subsequence (since the unit ball of a Hilbert space is weakly compact) in $H^{n}$. By uniqueness of limits, this implies that for all $t$, we have $w(\cdot, t) \in H^{n}$. 
We now want to show that $w \in C\left([0, T] ; H^{n}\right)$, and that $w$ solves the non-mollified equation.

To show that $w$ solves the non-mollified evolution equation, we integrate the mollified evolution equation in time:

$$
\begin{gathered}
w^{\epsilon}(x, t)=w_{0}(x)+\int_{0}^{t} 2 \mathcal{J}_{\epsilon}\left(\left(\left(\mathcal{J}_{\epsilon}\left(w^{\epsilon}\right)^{\beta}\right)(x, s)\right) \mathcal{J}_{\epsilon} w_{x x x}^{\epsilon}(x, s)\right) d s \\
+\int_{0}^{t}(12 \beta-6) \mathcal{J}_{\epsilon}\left(\left(\left(\mathcal{J}_{\epsilon} w^{\epsilon}\right)^{\beta-1}(x, s)\right)\left(\mathcal{J}_{\epsilon} w_{x}^{\epsilon}(x, s)\right) \mathcal{J}_{\epsilon} w_{x x}^{\epsilon}(x, s)\right) d s \\
+\int_{0}^{t}(\beta-1)(8 \beta-4) \mathcal{J}_{\epsilon}\left(\left(\left(\mathcal{J}_{\epsilon} w^{\epsilon}\right)^{\beta-2}(x, s)\right)\left(\mathcal{J}_{\epsilon} w_{x}^{\epsilon}(x, s)\right)^{3}\right) d s \\
+\int_{0}^{t} 2 \mathcal{J}_{\epsilon}\left(\left(\left(\mathcal{J}_{\epsilon} w^{\epsilon}\right)^{\beta}(x, s)\right) \mathcal{J}_{\epsilon} w_{x}^{\epsilon}(x, s)\right) d s
\end{gathered}
$$

where $t \in[0, T]$. Since $w^{\epsilon}$ converges uniformly to $w$ in $C\left([0, T] ; H^{n^{\prime}}\right)$ for any $0 \leq$ $n^{\prime}<n$, and since $n \geq 4$, we can pass to the limit in 61):

$$
\begin{aligned}
& w(x, t)=w_{0}(x)+ \\
& \quad \int_{0}^{t} 2 w^{\beta}(x, s) w_{x x x}(x, s)+(12 \beta-6) w^{\beta-1}(x, s) w_{x}(x, s) w_{x x}(x, s) d s \\
& \quad+\int_{0}^{t}(\beta-1)(8 \beta-4) w^{\beta-2}(x, s) w_{x}^{3}(x, s)+2 w^{\beta}(x, s) w_{x}(x, s) d s .
\end{aligned}
$$

This clearly implies that $w$ is a solution of the initial value problem.

All that remains is to demonstrate the highest regularity. We will begin by showing that $\|w\|_{H^{n}}$ is continuous as a function of time. We start by showing that the norm is right-continuous at $t=0$. By Fatou's Lemma, we have

$$
\|w(\cdot, 0)\|_{H^{n}}^{2} \leq \liminf _{t \rightarrow 0^{+}}\|w(\cdot, t)\|_{H^{n}}^{2} .
$$

From the energy inequality, however, we have

$$
\limsup _{t \rightarrow 0^{+}}\|w(\cdot, t)\|_{H^{n}}^{2} \leq\|w(\cdot, 0)\|_{H^{n}}^{2} .
$$

Therefore, the norm is right-continuous at $t=0$.

Given $t^{*} \in(0, T)$, we consider $t^{*}$ as a new initial time. We may solve the initial value problem starting from time $t^{*}$, with initial data $w\left(\cdot, t^{*}\right)$. By the previous argument, we see that this solution is right-continuous at time $t=t^{*}$. By the uniqueness theorem, we know that this solution starting from time $t^{*}$ and the previous solution, $w$, are the same. Therefore, $\|w\|_{H^{n}}$ is right-continuous at all $t \in$ $[0, T)$. Furthermore, all of the analysis we have performed works with time reversed: there is no feature of our estimates which requires time to move forward. Therefore, all of this analysis could be reversed, finding that $\|w\|_{H^{n}}$ is left-continuous at all $t \in(0, T]$.

To complete the proof, we use the fact that weak convergence together with convergence of the norm implies strong convergence. We have already shown convergence of the norm, so as the final step in our proof, we show that $w$ is weakly continuous in time. Let $\psi \in H^{-n}$ be given. Given $t^{*} \in[0, T]$, we want to show

$$
\left\langle\psi, w(\cdot, t)-w\left(\cdot, t^{*}\right)\right\rangle_{L^{2}} \rightarrow 0,
$$


as $t \rightarrow t^{*}$. Let $\delta>0$ be given. Let $K>0$ be such that for all $t \in[0, T],\|w(\cdot, t)\|_{H^{n}} \leq$ $K$. Let $n^{\prime}$ be given such that $0 \leq n^{\prime}<n$. Then, of course, $-n<-n^{\prime}$, and $H^{-n^{\prime}}$ is dense in $H^{-n}$. Therefore we can find $\psi_{\delta}$ such that

$$
\left\|\psi_{\delta}-\psi\right\|_{-n} \leq \frac{\delta}{3(1+K)} .
$$

This implies the following bound:

$$
\left|\left\langle\psi-\psi_{\delta}, w(\cdot, t)-w\left(\cdot, t^{*}\right)\right\rangle_{L^{2}}\right| \leq\left(\frac{\delta}{3(1+K)}\right)(2 K) \leq \frac{2 \delta}{3} .
$$

Now, since $w \in C\left([0, T] ; H^{n^{\prime}}\right)$, we can take $t$ close enough to $t^{*}$ such that

$$
\left\|w(\cdot, t)-w\left(\cdot, t^{*}\right)\right\|_{H^{n^{\prime}}} \leq \frac{\delta}{3\left(1+\left\|\psi_{\delta}\right\|_{-n^{\prime}}\right)} .
$$

This implies the following bound:

$$
\left|\left\langle\psi_{\delta}, w(\cdot, t)-w\left(\cdot, t^{*}\right)\right\rangle_{L^{2}}\right| \leq\left\|\psi_{\delta}\right\|_{-n^{\prime}}\left(\frac{\delta}{3\left(1+\left\|\psi_{\delta}\right\|_{-n^{\prime}}\right)}\right) \leq \frac{\delta}{3} .
$$

Putting this together, we find that we can take $t$ sufficiently close to $t^{*}$ to get

$$
\left|\left\langle\psi, w(\cdot, t)-w\left(\cdot, t^{*}\right)\right\rangle_{L^{2}}\right| \leq \delta .
$$

This implies that $w$ is weakly continuous in time. This completes the proof.

We sum up our results for the $K(2,2)$ equation in the following corollary:

Corollary 19. Let $n$ be an integer such that $n \geq 4$. Let $X$ be a periodic interval. Let $a_{0}>0$. Let $u_{0} \in H^{n}$ be such that $u_{0}(x)>a_{0}$ for all $x \in X$. Then there exists $T>0$ and $u \in C\left([0, T] ; H^{n}\right)$ such that for all $x \in X$ and for all $t \in[0, T]$, u satisfies $u(x, t)>a_{0} / 2$, and $u$ is the unique solution of the initial value problem (1) with $u(x, 0)=u_{0}(x)$.

Let $n^{\prime}$ be given such that $0 \leq n^{\prime}<n$, and let $\epsilon>0$ be given. There exists $\delta>0$ such that for any $v_{0} \in H^{n}$ such that $v_{0}(x)>a_{0}$ for all $x \in X$ and such that $\left\|v_{0}-u_{0}\right\|_{H^{n}} \leq \delta$, the solution, $v$, of the initial value problem (1) with $v(x, 0)=v_{0}(x)$ satisfies $v \in C\left([0, T] ; H^{n}\right)$, and

$$
\sup _{t \in[0, T]}\|u(\cdot, t)-v(\cdot, t)\|_{H^{n^{\prime}}}<\epsilon .
$$

\section{OTHER EXAMPLES OF QUASILINEAR EQUATIONS}

In this section, we give additional examples of quasilinear equations for which our method gives a short-time well-posedness result. Like (1), these are also equations in which the dispersive effect appears nonlinearly. The first additional example is the Harry Dym equation, and the other examples arise in numerical analysis of finite difference schemes.

4.1. Well-posedness of the Harry Dym Equation. The Harry Dym equation is

$$
u_{t}=u^{3} u_{x x x}
$$

This is a completely integrable equation [1] which has been shown to have applications in interfacial fluid dynamics [18. We consider this with the initial condition

$$
u(x, 0)=u_{0}(x) \text {. }
$$


As in the case of the $K(2,2)$ equation, we can show that the initial value problem for the Harry Dym equation is well-posed for $H^{4}$ initial data which is bounded away from zero. The argument is completely analogous to the $K(2,2)$ case, so we only present the essential step, which is the $H^{n}$ energy estimate. When providing all the details of the well-posedness proof, as we have done for the $K(2,2)$ equation, the energy estimate must in fact be carried out for a mollified version of the equation; in this section, we proceed informally, and present the energy estimate without first introducing mollifiers. Certainly, however, all of the details of the present argument can be carried out carefully as in the preceding section. We remark that, as in the case of the $K(2,2)$ equation, the average modified diffusion, $\bar{\delta}$, that we defined before is identically zero; in the case of the Harry Dym equation, this is because there is no $u_{x x}$ term present in 62 .

Two conserved quantities for the Harry Dym equation are the following:

$$
\int_{X} \frac{1}{u(x, t)} d x, \quad \int_{X} \frac{1}{u^{2}(x, t)} d x .
$$

One view of the energy estimate which we are about to perform is that we are generalizing the first of these. We can take the view that the first of these conserved quantities tells us that the $L^{2}$ norm of $u^{-1 / 2}$ is conserved by the evolution.

Let $n$ be an integer, such that $n \geq 4$. We make the choice $\beta=\frac{1}{n-\frac{1}{2}}$, following the same construction as used for (1) above. Note that, as before, this implies $\beta \in(0,1)$; furthermore, we also have $3 \beta \in(0,1)$. As before, we define $w=u^{\frac{1}{\beta}}$, so that $u=w^{\beta}$. From the previous section, we have the following relevant formulas:

$$
\begin{gathered}
u_{t}=\beta w^{\beta-1} w_{t}, \\
u_{x x x}=\beta(\beta-1)(\beta-2) w^{\beta-3} w_{x}^{3}+3 \beta(\beta-1) w^{\beta-2} w_{x} w_{x x}+\beta w^{\beta-1} w_{x x x} .
\end{gathered}
$$

Combining these, we find the evolution equation for $w$ :

$$
w_{t}=w^{3 \beta} w_{x x x}+3(\beta-1) w^{3 \beta-1} w_{x} w_{x x}+(\beta-1)(\beta-2) w^{3 \beta-2} w_{x}^{3} .
$$

We apply the operator $\partial_{x}^{n}$ to 64 :

(65) $\partial_{x}^{n} w_{t}=w^{3 \beta} \partial_{x}^{n+3} w+(3 \beta+3 \beta n-3) w^{3 \beta-1} w_{x} \partial_{x}^{n+2} w+F_{1}[w] \partial_{x}^{n+1} w+F_{2}[w]$,

where

$$
\begin{aligned}
F_{1}[w]=\left(\begin{array}{l}
n \\
2
\end{array}\right) \partial_{x}^{2}\left(w^{3 \beta}\right)+3(\beta-1) n\left(w^{3 \beta-1} w_{x}\right)_{x} & \\
& +3(\beta-1) w^{3 \beta-1} w_{x x}+3(\beta-1)(\beta-2) w^{3 \beta-2} w_{x}^{2},
\end{aligned}
$$

and

$$
\begin{aligned}
F_{2}[w]= & \sum_{k=3}^{n}\left(\begin{array}{l}
n \\
k
\end{array}\right)\left(\partial_{x}^{k} w^{3 \beta}\right)\left(\partial_{x}^{n-k+3} w\right)+\sum_{\ell=1}^{n}\left(\begin{array}{l}
n \\
\ell
\end{array}\right)\left(\partial_{x}^{\ell} w^{3 \beta-1}\right)\left(\partial_{x}^{n-\ell+1} w\right) w_{x x} \\
+3(\beta-1) & \sum_{k=2}^{n-1} \sum_{\ell=1}^{k}\left(\begin{array}{l}
n \\
k
\end{array}\right)\left(\begin{array}{l}
k \\
\ell
\end{array}\right)\left(\partial_{x}^{\ell} w^{3 \beta-1}\right)\left(\partial_{x}^{k-\ell+1} w\right)\left(\partial_{x}^{n-k+2} w\right) \\
& +\left(\sum_{k=1}^{s-1}\left(\partial_{x}^{k}\left(3 w^{3 \beta-2} w_{x}^{2}\right)\right)\left(\partial_{x}^{n+1-k} w\right)\right)+\partial_{x}^{n-1}\left(\left(\partial_{x} w^{3 \beta-2}\right) w_{x}^{3}\right) .
\end{aligned}
$$


Since $F_{1}[w]$ includes at most second derivatives of $w$, we can bound the $L^{\infty}$ norm of $F_{1}[w]$ in terms of $\|w\|_{H^{n}}$. Since $F_{2}[w]$ includes at most $n$-many derivatives of $w$, we can bound the $L^{2}$ norm of $F_{2}[w]$ in terms of $\|w\|_{H^{n}}$.

We proceed to the energy estimate. We define the energy, $E(t)$, to be

$$
E(t)=\frac{1}{2} \int_{X} w^{2}+\left(\partial_{x}^{n} w\right)^{2} d x
$$

Then, differentiating, we find the following:

$$
\frac{d E}{d t}=\int_{X} w w_{t}+\left(\partial_{x}^{n} w\right)\left(\partial_{x}^{n} w_{t}\right) d x
$$

Clearly, since $n \geq 4$, we can bound $\int_{X} w w_{t} d x$ in terms of $E$. Also, the contributions from $F_{1}$ and $F_{2}$ are bounded in terms of $E$.

The remaining piece that we must concern ourselves with is

$$
\int_{X}\left(\partial_{x}^{n} w\right) w^{3 \beta} \partial_{x}^{n+3} w+(3 \beta+3 \beta n-3)\left(\partial_{x}^{n} w\right) w^{3 \beta-1} w_{x} \partial_{x}^{n+2} w d x .
$$

We integrate the first term by parts:

$$
\int_{X}-\left(\partial_{x}^{n+1} w\right) w^{3 \beta} \partial_{x}^{n+2} w+(3 \beta n-3)\left(\partial_{x}^{n} w\right) w_{x} \partial_{x}^{n+2} w d x
$$

We recognize a perfect derivative in the first term, and we integrate both terms by parts:

$$
\int_{X}\left(\frac{3 \beta}{2}-3 \beta n+3\right) w^{3 \beta-1} w_{x}\left(\partial_{x}^{n+1} w\right)^{2}-(3 \beta n-3)\left(\partial_{x}^{n} w\right) w_{x x} \partial_{x}^{n+1} w d x .
$$

Since $\beta=\frac{1}{n-\frac{1}{2}}$, the first of these terms is identically zero. Performing one more integration by parts, we see that the final term is bounded in terms of the energy.

Theorem 20. Let $n$ be an integer such that $n \geq 4$. Let $X$ be a periodic interval. Let $a_{0}>0$. Let $u_{0} \in H^{n}$ be such that $u_{0}(x)>a_{0}$ for all $x \in X$. Then there exists $T>0$ and $u \in C\left([0, T] ; H^{n}\right)$ such that for all $x \in X$ and for all $t \in[0, T], u$ satisfies $u(x, t)>a_{0} / 2$, and $u$ is the unique solution of the initial value problem (62), (63).

Let $n^{\prime}$ be given such that $0 \leq n^{\prime}<n$, and let $\epsilon>0$ be given. There exists $\delta>0$ such that for any $v_{0} \in H^{n}$ such that $v_{0}(x)>a_{0}$ for all $x \in X$ and such that $\left\|v_{0}-u_{0}\right\|_{H^{n}} \leq \delta$, the solution, $v$, of the initial value problem (62) with $v(x, 0)=$ $v_{0}(x)$ satisfies $v \in C\left([0, T] ; H^{n}\right)$, and

$$
\sup _{t \in[0, T]}\|u(\cdot, t)-v(\cdot, t)\|_{H^{n^{\prime}}}<\epsilon .
$$

4.2. Effective equations for finite difference schemes. In the paper [10, Goodman and Lax found that a certain finite difference scheme for the KdV equation has as an modified equation

$$
u_{t}+u u_{x}+\frac{1}{6} \Delta^{2} u u_{x x x}=0,
$$

where $\Delta$ is the constant spatial step. Similarly, in studying a finite-difference scheme for a nonlocal dispersive equation, Zumbrun found the following modified equation 19]:

$$
u_{t}+\left(u^{2}\right)_{x}=-c_{2} a^{2}\left(u u_{x x}\right)_{x},
$$


where $c_{2}$ and $a$ are constants.

Slight variations of the arguments of the previous sections, which we will not repeat, imply that both of these are well-posed in $H^{4}$, for initial data bounded away from zero. The important point is that as for the $K(2,2)$ equation and the Harry Dym equation, the average modified diffusion for 66 and for (67) is identically zero.

\section{REMARKS ON SINGULARITY FORMATION}

We mention some further results related to singularity formation; these results hold for all of the quasilinear equations we have studied. These singularity formation results stem from theorems in [3] on positivity preservation for equations with the form

$$
u_{t}=u F[u]+u_{x} G[x]
$$

where $F$ and $G$ are operators which can include $u$ and derivatives of $u$. (We note that the paper 8 also contains a positivity preservation theorem for the $K(2,2)$ equation.) The following theorem is a version of Theorem 6 of [3], which is proved using ideas from [5]:

Theorem 21. Let $T>0$ and let $u \in C\left([0, T] ; H^{4}(X)\right)$ be a solution of (1), (62), (66), or (67). For each $t \in[0, T]$, let $m(t)=\inf _{x \in X} u(x, t)$. Then, for all $t \in[0, T]$, we have $\operatorname{sgn}(m(t))=\operatorname{sgn}(m(0))$.

The following corollary is an immediate consequence of this:

Corollary 22. Let $n \in \mathbf{N}$ with $n \geq 4$. Let $a_{0}>0$. Let $u_{0} \in H^{n}(X)$ be such that $u_{0}(x)>a_{0}$, for all $x \in X$. Let $u$ be the solution of the initial value problem for (1), (62), (66), or (67), with $u(x, 0)=u_{0}(x)$. Assume there exists $x_{*} \in X$ and $t_{*}>0$ such that $u\left(x_{*}, t_{*}\right)=0$. Then there exists $t_{* *} \in\left(0, t_{*}\right]$ such that

$$
\lim _{t \rightarrow t_{* *}^{-}}\|u(\cdot, t)\|_{4}=+\infty
$$

\section{REFERENCES}

[1] T. Akhunov. Local well posedness of dispersive systems in 1D. PhD thesis, University of Chicago, 2011.

[2] D. M. Ambrose, G. Simpson, J. D. Wright, and D. G. Yang. Ill-posedness of degenerate dispersive equations. Preprint, 2011.

[3] David M. Ambrose and J. Douglas Wright. Preservation of support and positivity for solutions of degenerate evolution equations. Nonlinearity, 23(3):607-620, 2010.

[4] Alina Chertock and Doron Levy. Particle methods for dispersive equations. J. Comput. Phys., 171(2):708-730, 2001.

[5] Adrian Constantin and Joachim Escher. Wave breaking for nonlinear nonlocal shallow water equations. Acta Math., 181(2):229-243, 1998.

[6] W. Craig, T. Kappeler, and W. Strauss. Gain of regularity for equations of KdV type. Ann. Inst. H. Poincaré Anal. Non Linéaire, 9(2):147-186, 1992.

[7] Walter Craig and Jonathan Goodman. Linear dispersive equations of Airy type. J. Differential Equations, 87(1):38-61, 1990.

[8] J. de Frutos, M. Á. López Marcos, and J. M. Sanz-Serna. A finite difference scheme for the $K(2,2)$ compacton equation. J. Comput. Phys., 120(2):248-252, 1995.

[9] Lawrence C. Evans. Partial differential equations, volume 19 of Graduate Studies in Mathematics. American Mathematical Society, Providence, RI, second edition, 2010.

[10] Jonathan Goodman and Peter D. Lax. On dispersive difference schemes. I. Comm. Pure Appl. Math., 41(5):591-613, 1988. 
[11] Martin Kruskal. Nonlinear wave equations. In Dynamical systems, theory and applications (Rencontres, Battelle Res. Inst., Seattle, Wash., 1974), pages 310-354. Lecture Notes in Phys., Vol. 38. Springer, Berlin, 1975.

[12] Doron Levy, Chi-Wang Shu, and Jue Yan. Local discontinuous Galerkin methods for nonlinear dispersive equations. J. Comput. Phys., 196(2):751-772, 2004.

[13] Yi A. Li, Peter J. Olver, and Philip Rosenau. Non-analytic solutions of nonlinear wave models. In Nonlinear theory of generalized functions (Vienna, 1997), volume 401 of Chapman \& Hall/CRC Res. Notes Math., pages 129-145. Chapman \& Hall/CRC, Boca Raton, FL, 1999.

[14] Andrew J. Majda and Andrea L. Bertozzi. Vorticity and incompressible flow, volume 27 of Cambridge Texts in Applied Mathematics. Cambridge University Press, Cambridge, 2002.

[15] Philip Rosenau and J.M. Hyman. Compactons: solitons with finite wavelength. Phys. Rev. Lett., (70):564-567, 1993.

[16] Francisco Rus and Francisco R. Villatoro. Padé numerical method for the Rosenau-Hyman compacton equation. Math. Comput. Simulation, 76(1-3):188-192, 2007.

[17] Francisco Rus and Francisco R. Villatoro. Self-similar radiation from numerical RosenauHyman compactons. J. Comput. Phys., 227(1):440-454, 2007.

[18] S. Tanveer. Evolution of Hele-Shaw interface for small surface tension. Philos. Trans. Roy. Soc. London Ser. A, 343(1668):155-204, 1993.

[19] Kevin Zumbrun. On a nonlocal dispersive equation modeling particle suspensions. Quart. Appl. Math., 57(3):573-600, 1999.

Department of Mathematics, Drexel University, 3141 Chestnut St., Philadelphia, PA 19104, USA

E-mail address: ambrose@math.drexel.edu

Department of Mathematics, Drexel University, 3141 Chestnut St., Philadelphia, PA 19104, USA

E-mail address: jdoug@math.drexel.edu 